\title{
Resistance to androgen receptor signaling inhibition does not necessitate development of neuroendocrine prostate
}

\section{cancer}

W. Nathaniel Brennen, ${ }^{1,2}$ Yezi Zhu, ${ }^{2}$ Ilsa M. Coleman, ${ }^{3}$ Susan L. Dalrymple, ${ }^{1}$ Lizamma Antony, ${ }^{1}$ Radhika A. Patel, ${ }^{3}$ Brian Hanratty, ${ }^{3}$ Roshan Chikarmane, ${ }^{1}$ Alan K. Meeker, ${ }^{1,2,4}$ S. Lilly Zheng, ${ }^{5}$ Jody E. Hooper, ${ }^{4}$ Jun Luo, ${ }^{2}$ Angelo M. De Marzo, ${ }^{1,2,4}$ Eva Corey, ${ }^{6}$ Jianfeng $\mathrm{Xu},{ }^{5}$ Srinivasan Yegnasubramanian,, ${ }^{1,4}$ Michael C. Haffner, ${ }^{3,7}$ Peter S. Nelson, ${ }^{3,6}$ William G. Nelson, ${ }^{1,2,4}$ William B. Isaacs, ${ }^{2}$ and John T. Isaacs ${ }^{1,2,4}$

'Department of Oncology, Sidney Kimmel Comprehensive Cancer Center (SKCCC), Johns Hopkins University, Baltimore, Maryland, USA. ${ }^{2}$ Department of Urology, James Buchanan Brady Urological Institute, Johns Hopkins University School of Medicine, Baltimore, Maryland, USA. ${ }^{3}$ Division of Human Biology, Fred Hutchinson Cancer Research Center, Seattle, Washington, USA. ${ }^{4}$ Department of Pathology, SKCCC, Johns Hopkins University, Baltimore, Maryland, USA. ${ }^{5}$ Program for Personalized Cancer Care, North Shore University Health System, Evanston, Illinois, USA. ${ }^{6}$ Department of Urology and 'Department of Pathology, University of Washington, Seattle, Washington, USA.

Resistance to AR signaling inhibitors (ARSis) in a subset of metastatic castration-resistant prostate cancers (mCRPCs) occurs with the emergence of $A R^{-}$neuroendocrine prostate cancer (NEPC) coupled with mutations/deletions in PTEN, TP53, and RB1 and the overexpression of DNMTs, EZH2, and/or SOX2. To resolve whether the lack of AR is the driving factor for the emergence of the NE phenotype, molecular, cell, and tumor biology analyses were performed on 23 xenografts derived from patients with $P C$, recapitulating the full spectrum of genetic alterations proposed to drive NE differentiation. Additionally, phenotypic response to CRISPR/Cas9-mediated AR KO in AR+ CRPC cells was evaluated. These analyses document that (a) ARSi-resistant NEPC developed without androgen deprivation treatment; (b) ARS in ARSi-resistant $\mathrm{AR}^{+} / \mathrm{NE}^{+}$double-positive "amphicrine" mCRPCs did not suppress NE differentiation; (c) the lack of AR expression did not necessitate acquiring a NE phenotype, despite concomitant mutations/deletions in PTEN and TP53, and the loss of RB1 but occurred via emergence of an $\mathrm{AR}^{-} / \mathrm{NE}^{-}$double-negative PC (DNPC); (d) despite DNPC cells having homogeneous genetic driver mutations, they were phenotypically heterogeneous, expressing basal lineage markers alone or in combination with luminal lineage markers; and (e) AR loss was associated with AR promoter hypermethylation in NEPCs but not in DNPCs.

Conflict of interest: The authors have declared that no conflict of interest exists.

Copyright: (c) 2021, Brennen et al. This is an open access article published under the terms of the Creative Commons Attribution 4.0 International License.

Submitted: December 10, 2020

Accepted: March 10, 2021

Published: April 22, 2021

Reference information: /CI Insight. 2021;6(8):e146827.

https://doi.org/10.1172/jici.

insight.146827.

\section{Introduction}

The normal adult human prostate is composed of a simple stratified epithelium, the homeostasis of which is maintained via adult stem/progenitor cell turnover producing a steady-state, self-renewing condition (1). In the human adult man, prostate epithelial stem cells undergo asymmetric division for self-renewal while producing progenitor cells with limited proliferative ability (2). The percentage of epithelial cells proliferating per day (i.e., $0.19 \% \pm 0.03 \%$ ) is remarkably low in the human adult benign prostate, which balances the equally low percentage of epithelial cells dying per day (3). During this steady-state maintenance condition, the turnover time (i.e., the time required to renew the epithelium) is $500 \pm 79$ days (3). Neither stem cells nor progenitor cells express AR protein; however, they require AR-dependent paracrine factors (i.e., andromedins) from the stroma for their proliferation but not survival $(4,5)$. It is proposed that a rare subset (i.e., $0.59 \%$ ) of adult prostate basal cells comprises the epithelial stem/progenitor cells, which coexpress the full spectrum of prostate epithelial cell markers (i.e., keratin 5 [KRT5], KRT6A, KRT8, KRT14, KRT18, and KRT19; the transcription factor p63; glutathione- $S$-transferase $\pi$ [GSTP1]), but not AR $(2,6)$. This is supported by the fact that although the growth fraction in basal cells based upon Ki67 expression is quite 
low (i.e., $1.65 \% \pm 0.12 \%$ positive), it is 12 -fold higher than the growth fraction in luminal cells (i.e., $0.14 \%$ $\pm 0.06 \%$ positive; Figure $1 \mathrm{~A}$; refs. 7,8 ). Consistent with this basal location for the proliferating epithelial stem/progenitor cells is the nuclear expression of c-MYC by $\mathrm{AR}^{-}$cells within this compartment in benign human glands (Figure 1B).

These basal adult progenitor cells differentiate into 1 of 3 lineage progeny (6-11). In the first lineage, progenitors at a very low frequency (i.e., <1\%) differentiate into proliferation-quiescent neuroendocrine (NE) cell progeny with a loss of expression of p63, GSTP1, and basal cell KRTs (KRT5, KRT6A, KRT14, and KRT19) without gaining expression of AR (9-13). Although the majority of these NE cells lack detectable expression of the highly prostate-specific transcription factor, HOXB13 (Figure 1C), a small subset (i.e., 17\%) expressed this protein at a low level equal to that expressed by basal cells. In contrast, these cells acquire a high expression of NE lineage markers, such as chromogranin A (CHGA; Figure 1C), CHG B (CHGB), and synaptophysin (SYP; refs. 9-13). In the second lineage, progenitors differentiate into basal cell progeny, which mature and maintain the expression of KRT5, KRT6A, KRT14, and KRT19; p63 (Figure 1D); and GSTP1 (Figure 1E), while losing the expression of KRT8 and KRT18 without gaining the expression of AR (6-13). In addition, these basal cells characteristically express the transcription factors SOX2 (Figure 1F; ref. 14), YAP-1 (15) and nerve growth factor receptor (NGFR, also known as p75; Figure 1D; ref. 16), coupled with low-to-moderate HOXB13 expression (Figure 1G; ref. 17), consistent with its expression being AR independent (18). In the third lineage, progenitors differentiated into luminal cell progeny, which maintained the expression of KRT8 and KRT18 while losing the expression of all other stem/progenitor markers (i.e., KRT5, KRT6A, KRT14, KRT19, and p63, Figure 1D; GSTP1, Figure 1E; SOX2, Figure 1F; YAP-1 and NFGR, Figure 1D; refs. 6-11, 15). In addition, they acquire the expression of AR (6) and prostate-specific membrane antigen (PSMA, also known as FOLH1; ref. 19), along with a 6-fold increase in HOXB13 (Figure $1, \mathrm{G}$ and $\mathrm{H}$; refs. 17, 20). AR transcriptional activity is not required, however, for commitment to luminal cell differentiation (21) because HOXB13 is not an AR target gene (18) and PSMA transcription is inhibited by AR (22). In contrast, AR expression and ligand occupancy are required for terminal luminal differentiation into a mature proliferation-quiescent secretory cell (21). This terminal differentiation is characterized by the gain of expression of AR-dependent prostate luminal cell lineage markers, such as NKX3.1, prostate-specific antigen (PSA, also known as $K L K 3)$, and hK2 $(K L K 2)$ with no expression of NE markers $(6,23)$ or the proliferation markers, Ki67 (Figure 1A) or c-Myc (Figure 1B).

During prostate carcinogenesis, molecular changes occur in prostate epithelial cells such that AR signaling (ARS) is subverted from a growth suppressor of c-Myc expression to a cell autonomous oncogenic stimulator of c-Myc expression and thus malignant growth (24-26). Due to this acquired oncogenic ARS addiction, androgen deprivation therapy (ADT) is the standard of care for metastatic castration-resistant prostate cancer (mCRPC). This is because ADT not only inhibits PC cell proliferation but also induces apoptotic cell death (4). Although initially responsive to such "castration therapy," metastatic cancer cells inevitably progress to a CR state given enough time and selective pressure (27). In the majority of cases, these mCRPC cells continue to express AR and their lethal growth is still stimulated by AR-dependent transcription despite greater than $90 \%$ suppression of serum androgen by ADT (27). These results validate that further disrupting $\mathrm{AR}$ function is a rational therapeutic approach for mCRPCs progressing on ADT.

Based upon this realization, next-generation ARS inhibitors (ARSis), such as abiraterone acetate (Abi), enzalutamide (Enza), and apalutamide, were developed and clinically documented to increase the survival of men with $\mathrm{mCRPC}$, progressing after first-line $\mathrm{ADT}$ and when given in combination with first-line ADT (27). Despite these advances, $\mathrm{mCRPC}$ remains a lethal disease due to the inevitable progression of these cancers to an ARSi-resistant state $(28,29)$. Approximately one third of these ARSi-resistant cancers are $\mathrm{AR}^{-}$(28). The proposed mechanism for this progression involves an initially $\mathrm{AR}^{+}$adenocarcinoma (ARPC) "losing" its luminal cell differentiation via the loss of AR activity (30-34). It has been proposed that such a loss of AR-dependent transcription enables "lineage plasticity," driving transdifferentiation of the initial ARPC to a more aggressive lethal $\mathrm{AR}^{-}$treatment-related NEPC phenotype (30-35).

Therefore, to interrogate the relationship between ARSi resistance and NE differentiation, 3 complementary approaches were taken. First, the growth characteristics and expression of basal versus luminal versus NE lineage markers were evaluated in a large series $(n=23)$ of previously characterized patient-derived xenografts (PDXs) in addition to several newly established PDXs, which collectively recapitulate the full spectrum of clinically important genetic alterations in mCRPC. Second, hypermethylation of the AR promoter as a putative mechanism for the loss of AR expression was evaluated. Third, the in vitro and 

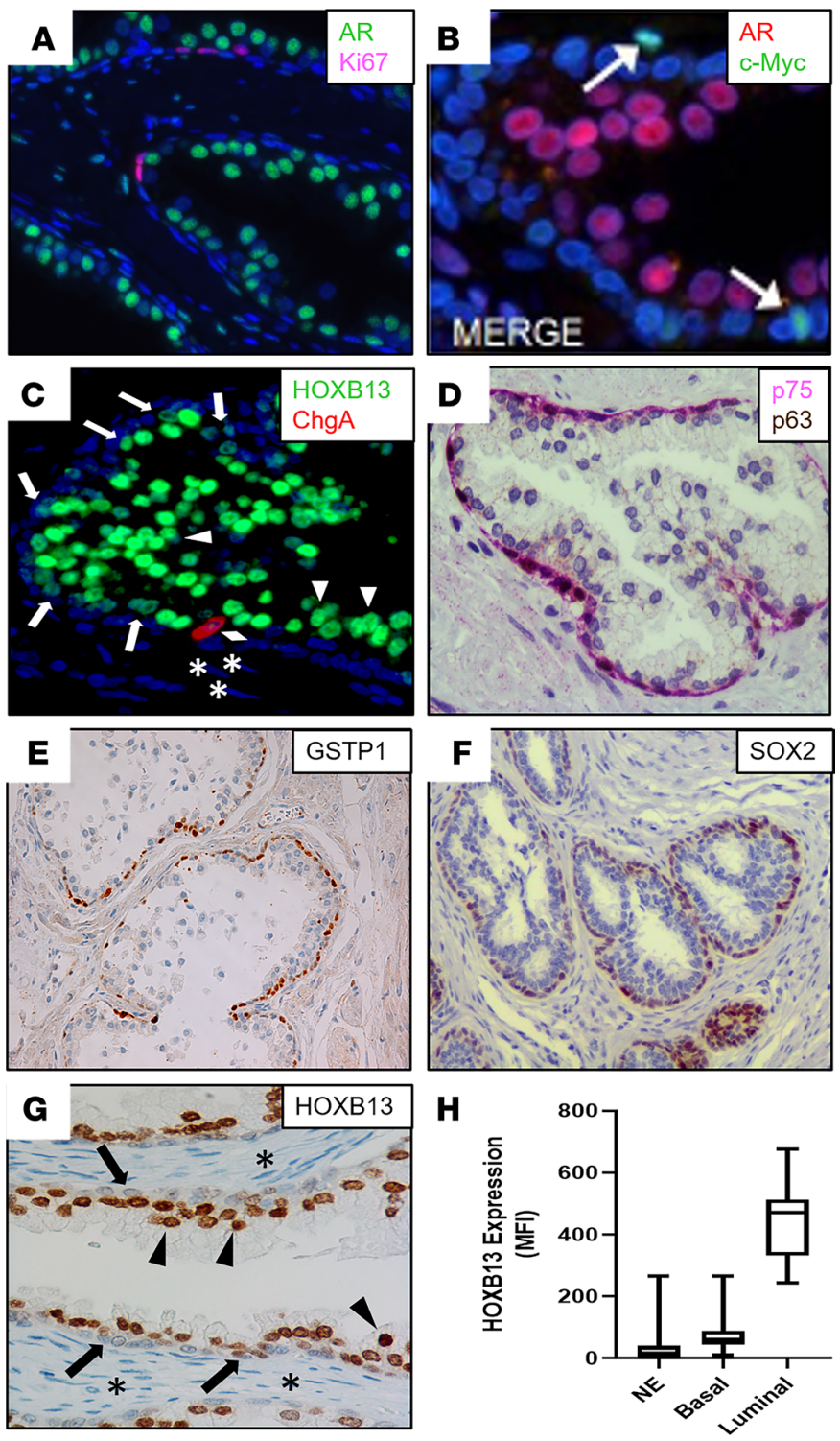

Figure 1. Lineage marker expression in the benign prostate. (A) IF staining of the AR (green) and Ki67 (pink), which documents that the majority of proliferation is restricted to the basal epithelial layer. Nuclei stained with DAPI (blue). (B) IF staining of c-Myc (green) and AR (red), documenting that the small subset of basal cells expressing c-Myc does not express AR. Nuclei stained with DAPI (blue). (c) IF staining of HOXB13 (green) and CHCA (red). Nuclei stained with DAPI (blue). Arrowheads indicate HOXB13-high luminal cells. Arrows indicate HOXB13-low basal cells. Diamond indicates $\mathrm{CHGA}^{+}$neuroendocrine cell. Asterisks indicate HOXB13- stromal cells (D) Dual IHC staining of NFGR (pink) and p63 (brown) identifies the basal layer. (E) IHC staining of GSTP1 (brown) in basal layer. (F) IHC staining of SOX2 in basal layer. (C) IHC staining of HOXB13. Arrowheads indicate HOXB13high luminal cells. Arrows indicate HOXB13-low basal cells. Asterisks indicate HOXB13- stromal cells. (H) Box plots indicate median and IQR range for the MFI of HOXB13 staining normalized to nuclear area in neuroendocrine ( $n$ $=97)$, basal $(n=97)$, and luminal cells $(n=24)$ of the normal prostate (whiskers $=\min / \mathrm{max}$ values). CHGA, chromogranin A; NGFR, nerve growth factor receptor.

in vivo growth characteristics versus marker expression of the ARPC LNCaP-95 (LN-95) mCRPC cells initially exhibiting AR activity were determined following CRISPR/Cas9 dependent elimination of total AR protein expression.

\section{Results}

Development of ARSi-resistant NEPC does not require prior ADT. The NCI-H660 cell line was established from a cervical lymph node metastasis from an untreated 63-year-old man diagnosed with small cell cancer of the prostate who presented with metastatic sites in the brain, liver, lymph nodes, subcutaneous tissue, bone, 
and bone marrow (36). The patient died 4 days after tissue harvest (i.e., 18 days after initial diagnosis) having never received ADT. The in vivo growth of H660 cells in adult male NSG hosts is ARSi resistant, because it grew equally well in intact or castrated hosts with a doubling time of $10 \pm 5$ days (Figure 2A), which was not affected by daily oral treatment with a therapeutically effective dose of either Abi or Enza. Histologically, this xenograft has been classified as a small-cell carcinoma (Figure 2B). It has a TP53 exon 9-11 deletion (37) and TMPRSS2-ERG fusion due to a homozygous intronic deletion (38), but expressed neither AR (Figure 2C) nor ERG (Figure 2D). It also did not express HOXB13 (Figure 2E; ref. 39), the basal cell markers GSTP1, KRT5, NGFR (Figure 2F), or p63 (Figure 2F). It did, however, uniformly express NE markers such as SYP (Figure 2G) and CHGA (Figure 2H). Thus, H660 is a "classic" ARSi-resistant NEPC, which developed without ADT treatment.

$A R$ does not suppress NE differentiation in amphicrine PC PDXs. LvCaP-2 is a newly described PC PDX derived from a liver metastasis obtained at rapid autopsy at John Hopkins from an ARSi-resistant patient with mCRPC (29). When adult male hosts bearing the LvCaP-2 PDX are castrated, the cancer stops growing for approximately 1 month before relapsing (29). Subsequent passage of a relapsing tumor in castrated hosts results in a variant, named LvCaP-2R, that grows equally well in intact and castrated hosts (doubling time of $10 \pm 3$ days versus $9 \pm 2$ days, respectively). The growth of $\mathrm{LvCaP}-2 \mathrm{R}$ in castrated mice is resistant to daily oral treatment with Abi or Enza (29). Histologically, LvCaP-2 and LvCaP-2R are high-grade adenocarcinomas, which genetically have a hemizygous loss-of-function (LOF) truncating mutation in TP53 (T211fs) and hemizygous deleterious mutation (R130Q) in PTEN with a loss of PTEN protein expression (29). Although they have WT $R B 1$, there is only limited focal expression of RB1 protein. In addition to expressing prostate-specific HOXB13 and luminal-specific, but not basal-specific, markers (Figure 3A), they expressed NE markers (Figure 3B). This is despite expressing AR at a 52-fold higher mRNA level (Figure 3A) with 11-fold higher nuclear localization of AR protein compared with normal prostate luminal cells (29). Importantly, AR and NE markers like SYP are coexpressed in the same cell (Figure 3, C-E). This AR is functional as documented by the expression (Figure $3 \mathrm{~F}$ ) and secretion of AR target proteins such as PSA (Supplemental Figure 1A; supplemental material available online with this article; https:// doi.org/10.1172/jci.insight.146827DS1). They also expressed REST and YAP1 (Figure 3A), the latter of which is a basal lineage marker, and neither of which is expressed in $\operatorname{NEPC}(15,40)$. These cancers thus represent "amphicrine" prostate carcinomas (AMPCs; i.e., $\mathrm{AR}^{+} / \mathrm{NE}^{+}$; refs. 41, 42). A similar coexpression of luminal and NE markers without basal marker expression occurs in the $\mathrm{AR}^{+} \mathrm{LuCaP}-77 \mathrm{CR} \mathrm{PDX}$, which is an ARSi-resistant AMPC variant of LuCaP-77 derived from a bone (femur) metastasis at rapid autopsy (43). These results document that ARS does not suppress NE differentiation in ARSi-resistant $\mathrm{AR}^{+} / \mathrm{NE}^{+}$ double-positive AMPCs and that NE differentiation can occur in the presence of ARSi-resistant AR signaling, resulting in ARSi-resistant $\mathrm{AR}^{+} / \mathrm{NE}^{+}$double-positive AMPCs.

$A R S i$ resistance in PDXs lacking AR expression does not necessitate NE differentiation. The BCaP-1 PDX was derived from a soft tissue metastasis adjacent to the right tibia obtained at rapid autopsy from a 63-year-old African American patient, who, at the time of initial presentation, had bone and lymph node metastases and an initial diagnostic prostate biopsy that was positive for carcinoma with a Gleason sum score of 9. Over the next year, the patient was treated with ADT followed by palliative external beam radiation of the bone before death but never received treatment with next-generation ARSis (Table 1 and Supplemental Figure 2A). At autopsy, 3 of the 4 metastatic lesions collected were completely negative for AR, PSA, and NKX3.1, including the bone metastasis used to establish the BCaP-1 PDX (Supplemental Table 1). In 1 of the collected metastases (i.e., bone - L4) and the localized prostate lesion, AR staining was heterogeneous (Supplemental Table 1). The expression of AR-dependent genes (PSA, NKX3.1, etc.) was consistent with the AR expression pattern in these lesions. In contrast, all 4 of the metastatic lesions collected from the patient in addition to the localized cancer in the prostate had PTEN-loss, RB1-loss, and mutated TP53 (i.e., genetic drivers), which are consistent with the PDX. Despite the patient never being exposed to ARSi treatment, the in vivo growth of BCaP-1 PDX is ARSi resistant because it grows equally well in intact or castrated hosts, with a doubling time of $20 \pm 5$ days (Figure 4A), which was not affected by additional treatment with either Abi or Enza. This is consistent with the fact that the metastatic lesion from which this PDX is derived was $\mathrm{AR}^{-}$. Histologically, this PDX is a high-grade carcinoma (Figure 4B). Based upon a combination of RNA-Seq, Western blotting, IHC staining, and targeted DNA sequencing analyses, Table 1 summarizes the most relevant characteristics of the BCaP-1 PDX. Consistent with the metastatic lesions in the patient, BCaP-1 uniformly expressed mutated TP53 (R175H; Figure 3A and 

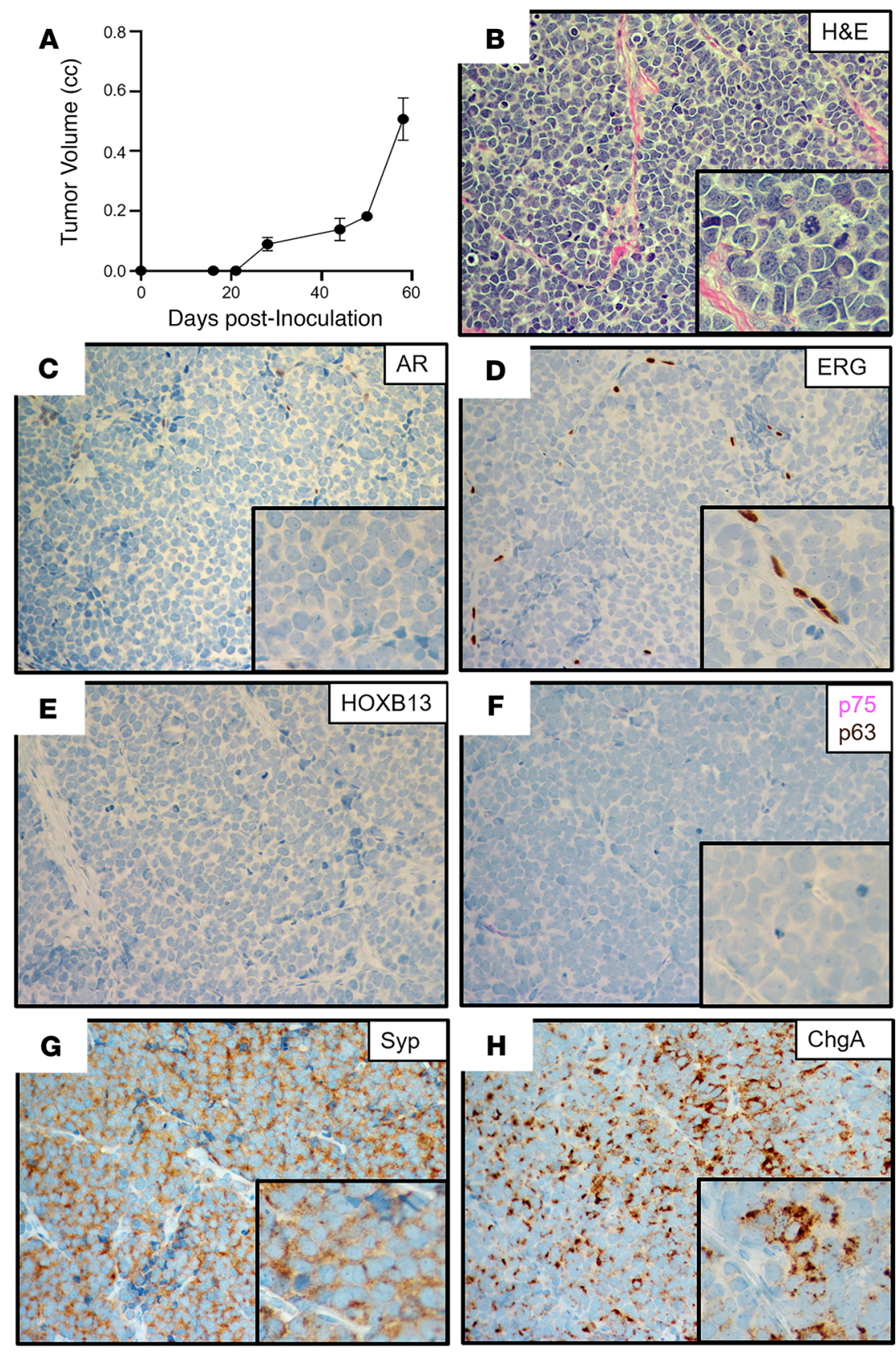

Figure 2. Characteristics of the NCI-H660 xenograft. (A) Growth rate in castrated NSG male mice $(n=5)$. (B) H\&E histology (original magnification, $\times 200$; inset [original magnification, $\times 400$ ]). (C-H) IHC (original magnification, $\times 200$ ) for (C) AR; (D) ERG (note positive staining in tumor endothelial cells); (E) HOXB13; (F) NFGR (pink) and p63 (brown) dual stain; (C) SYP; and (H) CHGA. NGFR, nerve growth factor receptor; SYP, synaptophysin; CHGA, chromogranin A.

Figure 4C) coupled with minimal expression of a mutated RB1 (P298fs) allele and loss of the other WT $R B 1$ allele (Figure 3A and Figure 4D). These cells uniformly lacked PTEN expression due to a homozygous deletion (Figure 3A), resulting in no detectable PTEN protein (Figure 4E). They also expressed a mutated CTNNB1 (S45F; Figure 3A), which was localized in the nucleus (Figure 4F), presumably activating CTNNB1-driven gene expression. BCaP-1 had a high expression of $c-M Y C$ and Ki67 (Figure 3A), with a high proportion of cells showing nuclear localization of these proteins (Figure $4, \mathrm{G}$ and $\mathrm{H}$ ). This is despite minimal expression of $A R$ or glucocorticoid receptor $(G R)$ mRNA (Figure 3A) and no expression of AR or GR protein. Consistent with the lack of AR protein, BCaP-1 did not express AR target genes like PSA and NKX3-1 that are characteristic of luminal cells (Table 1, Figure 3E, and Supplemental Figure 1A). However, they did uniformly express other markers characteristic of luminal cells, such as 
A Select Genes Expression Profile

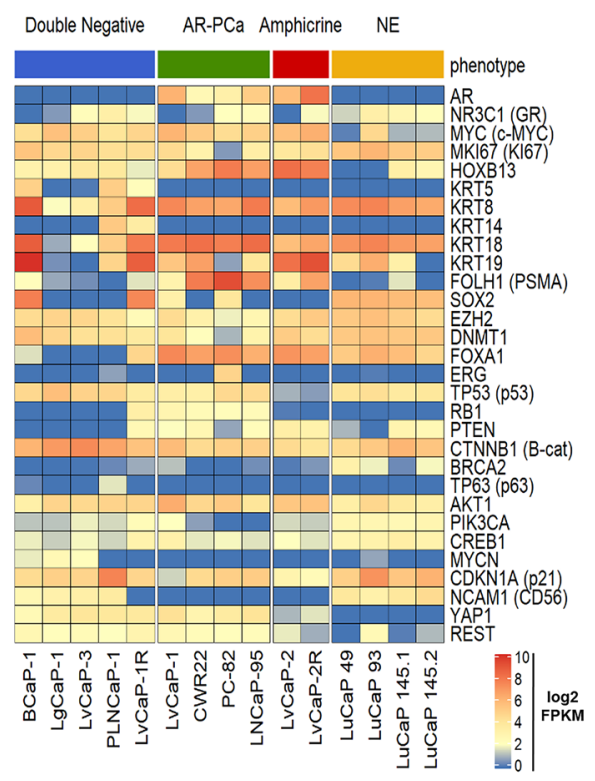

B
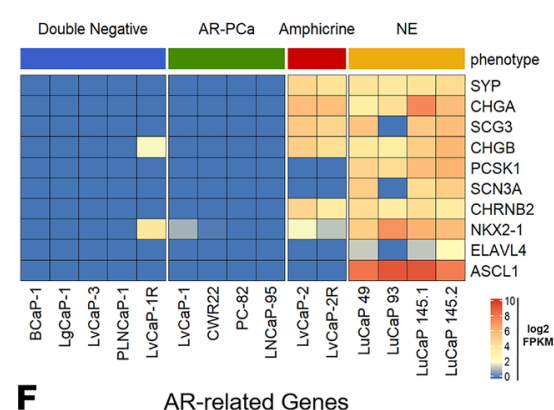

$\mathbf{F}$

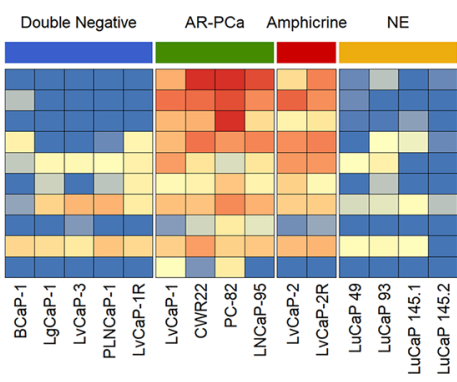

LvCaP-2R
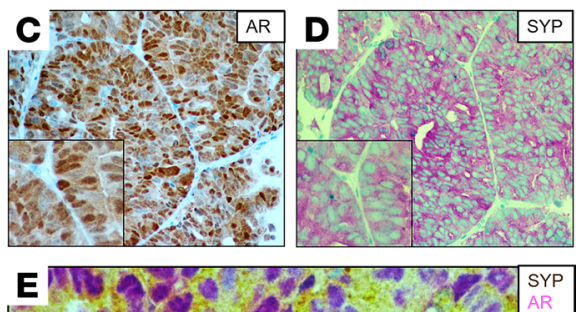

Figure 3. Gene expression in lethal mCRPC PDXs and amphicrine phenotype in LvCaP-2R. Lethal mCRPC PDXs analyzed for RNA expression of (A) select genes and (B) NE-associated genes. (C-E) IHC step-section of LvCaP-2R PDX stained for (C) AR (original magnification, $\times 200$ ), (D) SYP (original magnification, $\times 200$ ), and (E) dual staining (original magnification, $\times 400$ ) for AR (pink) and SYP (brown), documenting the coexpression of both markers in the same cell (i.e., amphicrine). (F) RNA-Seq analysis for AR-regulated genes in a panel of PDXs representing different phenotypes (e.g., DN, ARPC, amphicrine, and NE). mCRPC, metastatic castration-resistant prostate cancer; NE, neuroendocrine; PDXs, patient-derived xenografts; SYP, synaptophysin; DN, double-negative.

KRT8 and KRT18 (Figure 3A and Figure 4I), while heterogeneously expressing characteristic basal cell markers, such as KRT5 (Figure 3A and Figure 4J), GSTP1 (Figure 4K; SkCaP-1 shown in Figure 4L as a negative control for GSTP1 staining), NGFR (also known as p75 neurotrophin receptor; Figure 4M), and p63 (Figure 4M), coupled with a uniform expression of SOX2 (Figure 4N; refs. 14, 44, 45). Additionally, they heterogeneously expressed a moderate-to-high level of nuclear HOXB13 protein (Figure 4O). The retention of HOXB13 expression confirms its prostatic origin. This moderate-to-high level of HOXB13 expression in a subset of cells is significant because this is the level of nuclear expression characteristic of prostatic luminal cells (20) and is consistent with its expression being AR-independent (18). These cells also expressed FOXA1 (Figure 3A), which is an important coregulator of HOXB13 via its binding to a 37-bp regulatory element that activates the expression independent of AR transcriptional activity (18). This heterogeneous moderate-to-high expression is consistent with why this PDX had a lower level of HOXB13 mRNA (Figure 3A) and protein detected by Western blot (Supplemental Figure 1B than ARPCs. BCaP-1 lacked the expression of $E R G$ (Figure 3A) and the majority of NE-related genes (Figure 3B), including CHGA (Figure 4P). They also expressed REST (Figure 3A) and the basal marker YAP1 (Figure $3 \mathrm{~A})$, neither of which are expressed in $\operatorname{NEPC}(15,40)$. Collectively, these results document that, despite the lack of AR expression by BCaP-1, even when coupled with LOF mutations in PTEN and RB1, plus a putative gain-of-function mutation in TP53 together with the overexpression of DNMT1 and EZH2 (Figure $3 \mathrm{~A})$, these genetic change do not drive BCaP-1's differentiation into a NEPC. Rather, it is an example of an $\mathrm{AR}^{-} / \mathrm{NE}^{-}$double-negative (DN) PC (DNPC) heterogeneously composed of cells expressing both basal and luminal cell characteristics, suggestive that their cancer-initiating cell was an $\mathrm{AR}^{-}$progenitor cell whose malignant transformation did not require exposure to ARSis.

Three additional PDXs were established from a 65-year-old European American patient with a germline $B R C A 2$ mutation (Y2215fs) who underwent resection for a noninvasive urothelial carcinoma of the bladder a year before having a prostate biopsy that was positive for Gleason $9 \mathrm{PC}$ with perineural invasion. Over the next 3 years, the patient had a radical prostatectomy for locally advanced disease (i.e., extraprostatic extension, seminal vesicle invasion, lymphatic invasion), followed by ADT, external beam radiation, taxane chemotherapy, and olaparib before undergoing a rapid autopsy upon his death (Supplemental Figure 2B). 
Table 1. Phenotypic and growth characteristics of a lethal mCRPC PDX series

\begin{tabular}{|c|c|c|c|c|c|c|}
\hline & BCaP-1 & LvCaP-3 & LgCaP-1 & PLNCaP-1 & LvCaP-1 & LvCaP-1R \\
\hline Tissue of origin & Bone met & Liver met & Lung met & Peripancreatic LN met & Liver met & LvCaP-1 \\
\hline $\begin{array}{l}\text { Patient treatment } \\
\text { history }\end{array}$ & $\begin{array}{l}\text { ADT and bone } \\
\text { radiation }\end{array}$ & $\mathrm{ADT}$, radiation & , chemotherapy, and & d PARP inhibitor & $\begin{array}{l}\text { ADT, taxane, } \\
\text { radiation, } \\
\text { abiraterone, } \\
\text { etoposide, and } \\
\text { cisplatin }\end{array}$ & \\
\hline Histology & $\begin{array}{l}\text { High-grade } \\
\text { carcinoma }\end{array}$ & $\begin{array}{l}\text { High-grade } \\
\text { carcinoma }\end{array}$ & $\begin{array}{l}\text { High-grade } \\
\text { carcinoma }\end{array}$ & High-grade carcinoma & $\begin{array}{l}\text { High-grade } \\
\text { carcinoma }\end{array}$ & $\begin{array}{c}\text { High-grade } \\
\text { carcinoma with } \\
\text { pleomorphic giant } \\
\text { cell features }\end{array}$ \\
\hline $\begin{array}{l}\text { In vivo growth } \\
\text { response to } A D T\end{array}$ & No & No & No & No & Yes & No \\
\hline $\begin{array}{l}\text { Xenograft doubling } \\
\text { time }\end{array}$ & $\begin{array}{c}20 \pm 5 \text { days } \\
\text { (intact or castrate } \\
\text { host) }\end{array}$ & $\begin{array}{c}31 \pm 3 \text { days } \\
\text { (intact or castrate } \\
\text { host) }\end{array}$ & $\begin{array}{c}18 \pm 2 \text { days } \\
\text { (intact or castrate } \\
\text { host) }\end{array}$ & $\begin{array}{c}44 \pm 5 \text { days } \\
\text { (intact or castrate } \\
\text { host) }\end{array}$ & $\begin{array}{l}12 \pm 2 \text { days } \\
\text { (intact host) }\end{array}$ & $\begin{array}{c}14 \pm 5 \text { days } \\
\text { (castrate host) }\end{array}$ \\
\hline Ki67 expression & $67 \pm 8 \%$ & $59 \pm 12 \%$ & $51 \pm 7 \%$ & $64 \pm 11 \%$ & $75 \pm 8 \%$ & $59 \pm 21 \%$ \\
\hline AR expression & WT, no expression & WT, no expression & WT, no expression & WT, no expression & WT, expressed & WT, no expression \\
\hline HoxB13 expression & Yes (sporadic) & Yes (sporadic) & Yes (sporadic) & Yes (sporadic) & Yes (sporadic) & Yes (sporadic) \\
\hline p53 expression & $\begin{array}{l}\text { GOF mutation } \\
\text { (R175H) }\end{array}$ & $\begin{array}{l}\text { GOF mutation } \\
\text { (R282W) }\end{array}$ & $\begin{array}{l}\text { GOF mutation } \\
\text { (R282W) }\end{array}$ & $\begin{array}{l}\text { GOF mutation } \\
\text { (R282W) }\end{array}$ & $\begin{array}{l}\text { GOF mutation } \\
\text { (R248Q) }\end{array}$ & $\begin{array}{l}\text { GOF mutation } \\
\text { (R248Q) }\end{array}$ \\
\hline PTEN expression & $\begin{array}{l}\text { Negative, } \\
\text { homozygous } \\
\text { deletion }\end{array}$ & $\begin{array}{l}\text { Negative, } \\
\text { homozygous } \\
\text { deletion }\end{array}$ & $\begin{array}{l}\text { Negative, } \\
\text { homozygous } \\
\text { deletion }\end{array}$ & $\begin{array}{l}\text { Negative, homozygous } \\
\text { deletion }\end{array}$ & $\begin{array}{l}\text { Negative, } \\
\text { homozygous LOF } \\
\text { mutation (V317fs) }\end{array}$ & $\begin{array}{c}\text { Negative, } \\
\text { homozygous LOF } \\
\text { mutation (V317fs) }\end{array}$ \\
\hline c-MYC expression & $82 \% \pm 13 \%$ & $90 \% \pm 21 \%$ & $83 \% \pm 15 \%$ & $73 \% \pm 16 \%$ & $70 \% \pm 9 \%$ & $59 \% \pm 16 \%$ \\
\hline Rb expression & $\begin{array}{l}\text { No, mutated } \\
\text { (P298fs) }\end{array}$ & No, WT & No, WT & No, WT & Yes, WT & Yes, WT \\
\hline$\beta$-Catenin expression & $\begin{array}{l}\text { Yes, nuclear mutant } \\
\text { (S45F) }\end{array}$ & Yes, WT & Yes, WT & Yes, WT & Yes, WT & Yes, WT \\
\hline BRCA2 status & WT & $\begin{array}{l}\text { LOF mutated } \\
\text { (Y2215fs) }\end{array}$ & $\begin{array}{l}\text { LOF mutated } \\
\text { (Y2215fs) }\end{array}$ & LOF mutated (Y2215fs) & WT & WT \\
\hline ERG expression & No & No & No & No & No & No \\
\hline CK5 expression & Yes & Focal & Rare foci & Yes & No & No \\
\hline p63 expression & Yes (sporadic) & Yes (sporadic) & Yes (sporadic) & Yes (sporadic) & No & Yes (sporadic) \\
\hline NGFR expression & Yes (focal) & Yes (focal) & Yes (focal) & Yes (focal) & No & Yes (sporadic) \\
\hline GSTP1 expression & Yes & Yes & Yes & Yes & No & No \\
\hline SOX2 expression & Yes & No & No & No & Yes & Yes \\
\hline CK18 expression & Yes & Yes & Yes & Yes & Yes & Yes \\
\hline NKX3.1 expression & No & No & No & No & Yes & No \\
\hline PSA expression & No & No & No & No & Limited focal & No \\
\hline PSMA expression & Low focal & No & No & No & Limited focal & Limited focal \\
\hline CHGA expression & Rare focal & No & No & No & No & No \\
\hline SYP expression & No & No & No & No & No & No \\
\hline
\end{tabular}

mCRPC, metastatic castration-resistant prostate cancer; PDX, patient-derived xenograft; ADT, androgen deprivation therapy; met, metastasis; NCFR, nerve growth factor receptor; GOF, gain of function; LOF, loss of function; CHCA, chromogranin A; SYP, synaptophysin.

All metastatic lesions collected from this patient had PTEN-loss and RB1-loss, in addition to mutated TP53 (Supplemental Table 1). From this autopsy, 3 independent PDXs were established from a liver metastasis (LvCaP-3), a lung metastasis (LgCaP-1), and a peripancreatic lymph node metastasis (PLNCaP-1). The in vivo growth of each of these PDXs was ARSi resistant, as documented by the fact that each grew equally well in intact versus castrate hosts and was not affected by the addition of treatment with either Abi or Enza. Interestingly, though derived from the same patient with lethal mCRPC, these 3 ARSi-resistant PDXs had different in vivo growth rates (Table 1 and Figure 5A), despite the fact that all 3 PDXs are histologically high-grade carcinomas (Figure 5B, Figure 6A, and Figure 7A).

Table 1 summarizes the most relevant characteristics of these 3 additional ARSi-resistant PDX models. All 3 of these additional PDXs lacked the expression of ERG (Figure 3A) and NE markers, 
A

$\underline{\mathrm{BCaP}-1}$
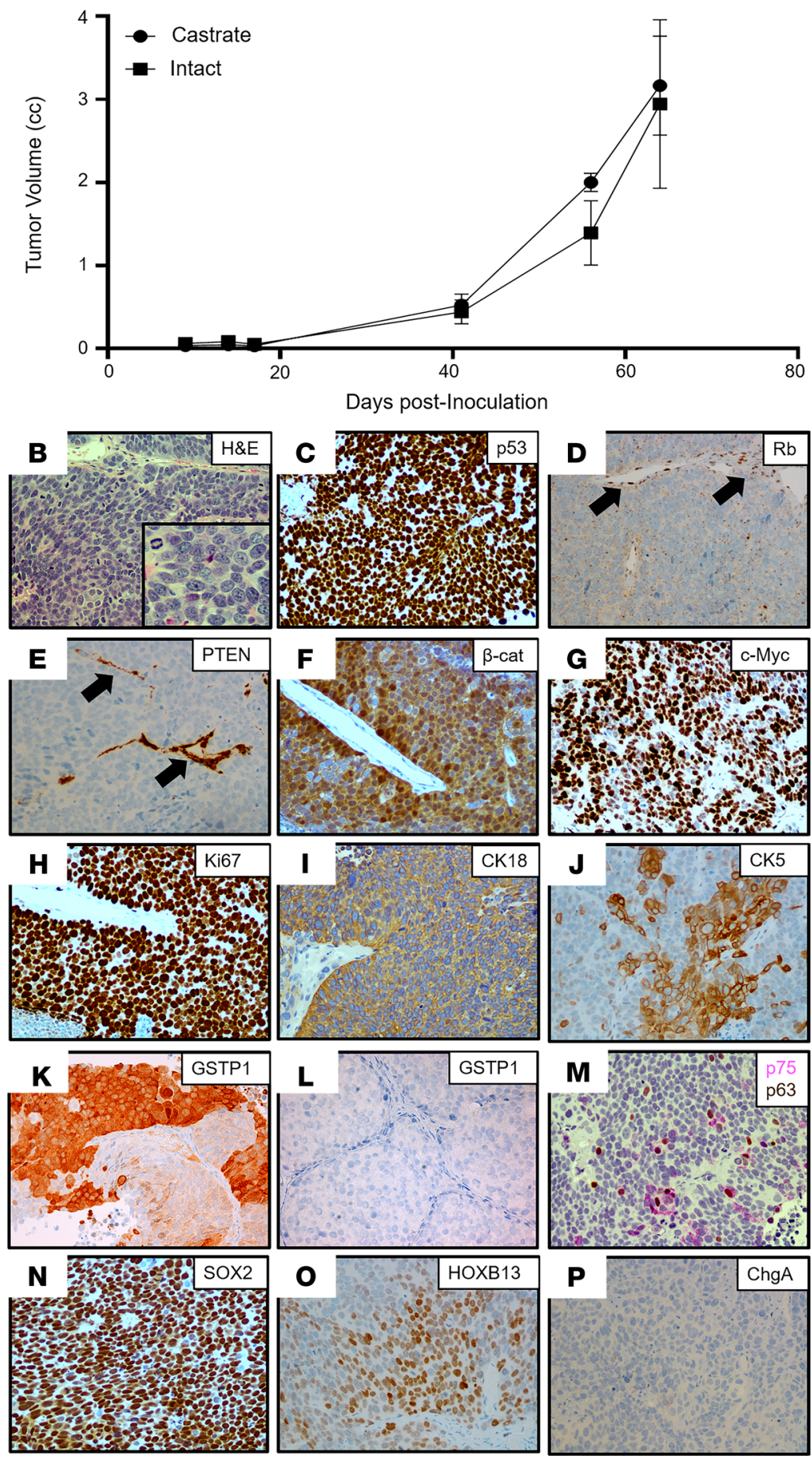

Days post-Inoculation
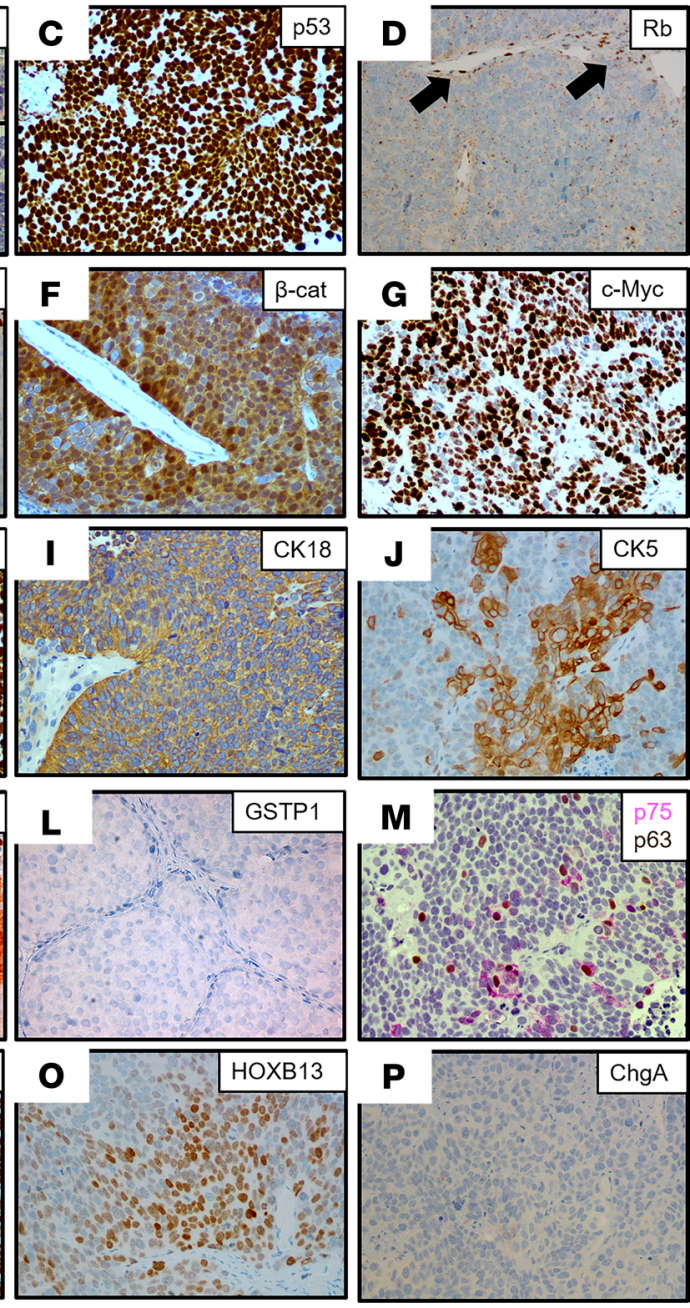

Figure 4. Characteristics of the BCaP-1 PDX. (A) Growth rate in intact vs. castrated NSG male mice $(n=5)$. (B) H\&E histology (original magnification, $\times 200$; inset [original magnification, $\times 400]$ ). (C-P) IHC (original magnification, $\times 200$ ) for (C) p53; (D) Rb (note that endothelial cell nuclei are an internal positive control for staining [black arrows]); (E) PTEN (note that endothelial cells are an internal positive control for staining [black arrows]); (F) $\beta$-catenin; (C) c-MYC; (H) Ki67; (I) CK18; (J) focal CK5; (K) CSTP1 in BCaP-1 (positive); (L) GSTP1 in $\mathrm{SkCaP}-1$ (negative control); (M) dual staining for p75 (pink) and p63 (brown); (N) Sox2; (0) HOXB13; and (P) CHGA. PDX, patient-derived xenograft; CHCA, chromogranin $A$.

including CHGA, CHGB, and SYP (Figure 3B), with minimal expression of either mutated $B R C A 2$ (Y2215fs) (Figure 3A) or mutated TP53 (R282W), and no expression of PTEN due to homozygous deletion. In addition, they minimally expressed $R B 1$ mRNA with no detectable nuclear expression of RB1 protein (Figure $3 \mathrm{~A}$ and Table 1). None of the cancer cells in these 3 PDXs express AR protein, thus explaining why they are resistant to ARSis. The tumors had a high proportion $(>50 \%)$ of cells with nuclear staining for c-MYC (Table 1, Figure 5C, and Figure 7B) and Ki67 (Table 1 and Figure 5D). They did not express KLK3 (PSA), FOLH1 (PSMA), or NKX3.1 (Table 1, Figure 3, A and E, and Supplemental Figure 1A). Again, similar to $\mathrm{AR}^{-} \mathrm{BCaP}-1$ cells, the fact that there was no expression of PSA or NKX3.1 in LvCaP-3, LgCaP-1, or PLNCaP-1 cells is predictable because these are known AR target genes. This is despite the fact that these cells expressed GR (Figure 3A). All 3 heterogeneously expressed a moderate-to-high level of nuclear HOXB13 protein (Figure 5E, Figure 6B, and Figure 7C). Again, this cellular heterogeneity is consistent with these PDXs having a lower level of HOXB13 mRNA (Figure 3A) and protein detected by Western blot (Supplemental Figure 1B) than ARPCs. The retention of HOXB13 expression in all 3 of these PDXs confirms their prostatic origin. Unlike BCaP-1 cells, however, HOXB13 expression in all 3 of these latter PDXs was independent of FOXA1 because they essentially had no expression of this transcription factor (Figure 3A).

All 3 of these PDXs retained the expression of luminal characteristic KRT8 (Figure 3A, Figure 6C, and Figure $7 \mathrm{D}$ ) and KRT18 (Figure 3A). Interestingly, although neither LvCaP-3 nor LgCaP-1 express basal characteristic KRT5 or KRT14, PLNCaP-1 coexpressed KRT5, KRT8, KRT14, KRT18, and KRT19 (Figure 3A). They also all uniformly expressed the basal marker GSTP1 (Figure 5F, Figure 6D, and Figure 7E), in addition to other basal characteristic markers such as NGFR focally and p63 sporadically (Figure 5G, Figure 6, E and F, and Figure 7F). Thus, despite the lack of AR and RB1 expression coupled with mutations in PTEN and TP53 and the overexpression of DNMT1 and EZH2 (Figure 3A), they were not NEPCs. Again, they expressed REST and the basal marker YAP1 (Figure 3A), which are not expressed in NEPC $(15,40)$. Thus, these PDXs are again examples of $\mathrm{AR}^{-} / \mathrm{NE}^{-} \mathrm{DNPC}$ with heterogeneous basal and luminal cell characteristics. 
Progression of ARPC PDX to ARSi resistance. These PDX models document that the lack of AR-dependent transcription in $\mathrm{AR}^{-} \mathrm{PC}$ cells does not necessitate differentiation into NEPC, even when combined with LOF/expression of PTEN, RB1, and p53. A possible explanation is that the cancer-initiating cells in these DN-PDXs are derived from transformed progenitor cells that never expressed AR and thus are unresponsive to AR-targeted therapy. Clearly, however, the majority of mCRPCs express AR. This raises the issue of whether the subset of ARPCs that lose AR expression in their progression to ARSi resistance induces lineage transdifferentiation into NEPC.

To test this possibility, another newly derived PDX, LvCaP-1, was used as a model system. LvCaP-1 was derived from a liver metastasis obtained at rapid autopsy from a 64-year-old European American patient who was treated over a 17-year period starting with a radical prostatectomy (Gleason sum 8) and then a PC vaccine (GVAX), followed by ADT, docetaxel plus strontium-89, external beam radiation, Abi, etoposide, and cisplatin (Supplemental Figure 2C; ref. 46). Similar to the radical prostatectomy specimen, all metastatic lesions collected from this patient at the time of autopsy were $\mathrm{AR}^{+}$and $\mathrm{NKX} 3-1^{+}$, in addition to having PTEN-loss and mutated TP53 (Supplemental Table 1). The most relevant characteristics of this PDX are summarized in Table 1. Histologically, LvCaP-1 is a high-grade adenocarcinoma (Figure 8A). Similar to the original patient-derived liver metastasis (46), this PDX had a 20-fold amplification of the $A R$ gene locus and a 64-fold higher level of WT $A R$ mRNA compared with localized PC (Figure 3A). Essentially, all LvCaP-1 cells exhibited high nuclear staining of AR-FL (Figure 8B). A high proportion (>70\%) of cells expressed c-MYC and Ki67 (Figure 3A). LvCaP-1 expressed NKX3-1, HOXB13 (mutated G84E), FOLH1, KLK2, and KLK3 (Figure 3, A and E, and Figure 8C), but with only a low level of PSA secretion (i.e., serum PSA of $1.4 \pm 0.4 \mathrm{ng} / \mathrm{mL} / \mathrm{g}$ tumor; Supplemental Figure 1A). This is coupled with the expression of mutated TP53 (R248Q; Figure 8D), SOX2, mutated SPOP (F133L), and mutated PTEN (V317fs), but with no expression of NE markers like $C H G A, C H G B$, and $S Y P$ (Figure 3, A and B). LvCaP-1 also did not express basal cell markers like GSTP1 (Figure 8E), p75 (Figure 8F), or p63 (Figure 8F).

When intact mice bearing LvCaP-1 PDXs growing with a $12 \pm 2$ day doubling time were castrated, the cancers regressed by greater than $90 \%$ over the next 80 days before relapsing (Figure $8 \mathrm{G}$ ), documenting that LvCaP-1 is an androgen-responsive ARPC. A relapsing tumor was serially passaged in castrated male mice to produce the LvCaP-1R PDX, which grows with a doubling time of $15 \pm 5$ days in castrated hosts (Figure $8 \mathrm{H}$ ). Oral treatment with effective daily doses of either Abi or Enza (29) has no effect upon the growth of LvCap$1 \mathrm{R}$ in castrated male hosts, documenting its ARSi resistance. Histologically, the LvCaP-1R is a high-grade carcinoma with focal pleomorphic giant cell features (Figure 8I). LvCaP-1R retains a 20-fold amplification of the $A R$ gene. Despite this amplification, LvCaP-1R had minimal expression of $A R$ mRNA (Figure $3 \mathrm{~A}$ ) and no detectable AR protein (Figure 8J). This PDX also lacked detectable expression of AR-dependent NXK3.1 and PSA, but retained a large proportion of cells expressing c-MYC and Ki67 (Figure 3A and Figure 8K). Like the parental LvCaP-1 PDX, it also retained mutated TP53 (R248Q), mutated PTEN(V317fs), mutated SPOP (F133L), and mutated ATRX (Table 1 and Figure 3A) and a uniform expression of basal marker SOX2 (Figure 8L). In addition, there was no gain of NE marker expression, including $C H G A, C H G B$, and $S Y P$ (Table 1 and Figure 3B). However, it did gain heterogeneous expression of basal markers like KRT5, KRT14, p63 (Figure $8 \mathrm{M}$ ), and NGFR (Figure 8, N and O), but not GSTP1 (Figure 8P). Importantly, it retained heterogeneous moderate-to-high expression of both the AR-independent HOXB13 (Figure 8Q) and the FOLH1 genes (Figure 3, A and E, and Supplemental Figure 1B), which are characteristic of luminal cells. These results document that the lack of AR expression by ARSi-resistant LVCaP-1R resulted in the acquisition of an $\mathrm{AR}^{-} / \mathrm{NE}^{-} \mathrm{DN}$ phenotype with heterogeneous expression of both basal and luminal cell markers, but lacked NE differentiation.

Response to acute loss of AR by ARSi-resistant PC cells. To directly test whether the acute loss of ARS can drive NE transdifferentiation of ARSi-resistant ARPC cells, a molecular approach was taken using the ARSi-resistant LN-95 PC cell line as a model. This cell line is a variant of LNCaP, produced by long-term in vitro growth in charcoal-stripped FBS (CS-FBS) media containing a low level of androgen (47). ARSi resistance is documented by the fact that both its in vitro and in vivo growth are resistant to Enza (29). This in vivo ARSi resistance is not due to intratumoral synthesis of androgens, as documented by the fact that serial passaging of LN-95 in castrated NSG hosts results in equivalent levels of intratumoral androgen as seen in patients treated with ARSi (i.e., Abi/Enza; refs. 48, 49). Importantly, we have documented previously that such ARSi resistance is not associated with a NE morphology, but that LN-95 remains a poorly differentiated ARPC whose growth (i.e., high Ki67 positivity) is dependent upon AR expression and signaling even when grown in a castrated host (Figure 9A, top; ref. 29). 
A

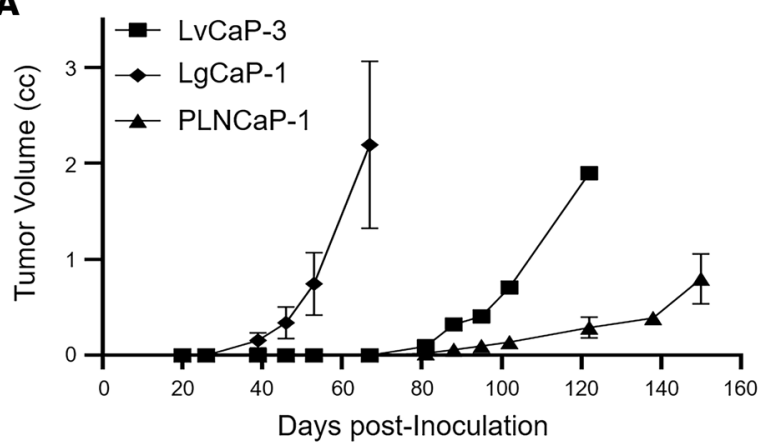

LvCaP-3
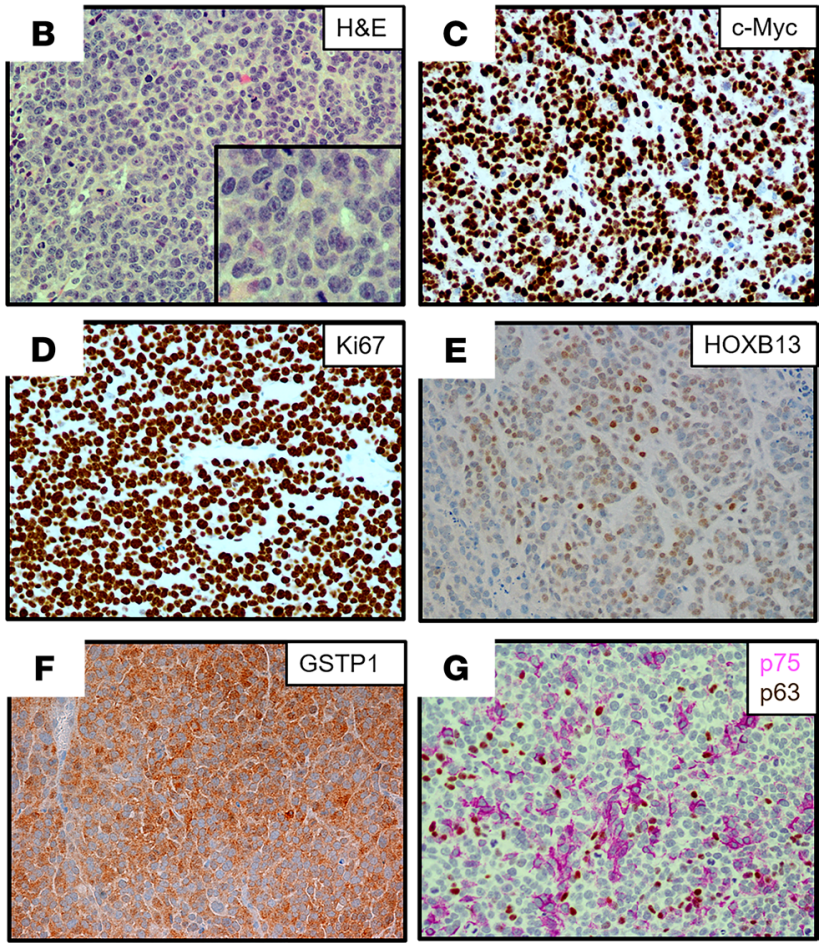

Figure 5. Characteristics of the LvCaP-3 PDX. (A) Comparative growth rate in castrated NSG male mice $(n=5)$ of LvCaP-3, LgCaP-1, and PLNCaP-1. (B) H\&E histology (original magnification, $\times 200$; inset [original magnification, $\times 400$ ]). (C-F) IHC (original magnification, $\times 200$ ) for (C) C-MYC, (D) Ki67, (E) HOXB13, and (F) GSTP1. (C) Dual staining for NGFR (pink) and p63 (brown). PDX, patient-derived xenograft; NGFR, nerve growth factor receptor.

Based upon this validation, CRISPR/Cas9 editing was used to delete the AR in LN-95 cells. Multiple clones were obtained in which both full-length and AR-V7 were simultaneously knocked out (i.e., total AR KO; Figure 9, B and C). The in vitro growth of these total AR-KO clones was slower than the parental AR-expressing LN-95 cells in CS-FBS media (Figure 9D). Importantly, these AR-KO clones did not acquire NE (i.e., dendritic) morphology in vitro (Supplemental Figure 3), nor did they upregulate NE markers like SYP, CHGA, or CHGB (Figure 9E) or gain the expression of basal markers like KRT5, KRT14, TP63, NGFR, or GSTP1 (Figure 9E). They did, however, downregulate the expression of AR target genes like NKX3-1, TMPRSS2, KLK2, and KLK3 (Figure 9F and Supplemental Figure 1C). Despite the loss of $\mathrm{AR}$ expression, they retained a high proportion of $\mathrm{Ki} 67^{+}$cells while remaining a high-grade carcinoma histologically in vivo (Figure 9A, bottom). These AR-KO clones, however, grew at a rate that was significantly slower $(P<0.05)$ than the parental AR-expressing LN-95 cells in castrated male hosts (Figure 9G). Importantly, the AR-KO clones retained the same high expression of AR-independent luminal genes (e.g., KRT8, KRT18, HOXB13, FOLH1) as the parental LN-95 cells despite the loss of AR expression (Figure 9F). These results document that AR is not suppressing the expression of NE or basal markers in the parental LN-95 ARSi-resistant ARPC cells. 


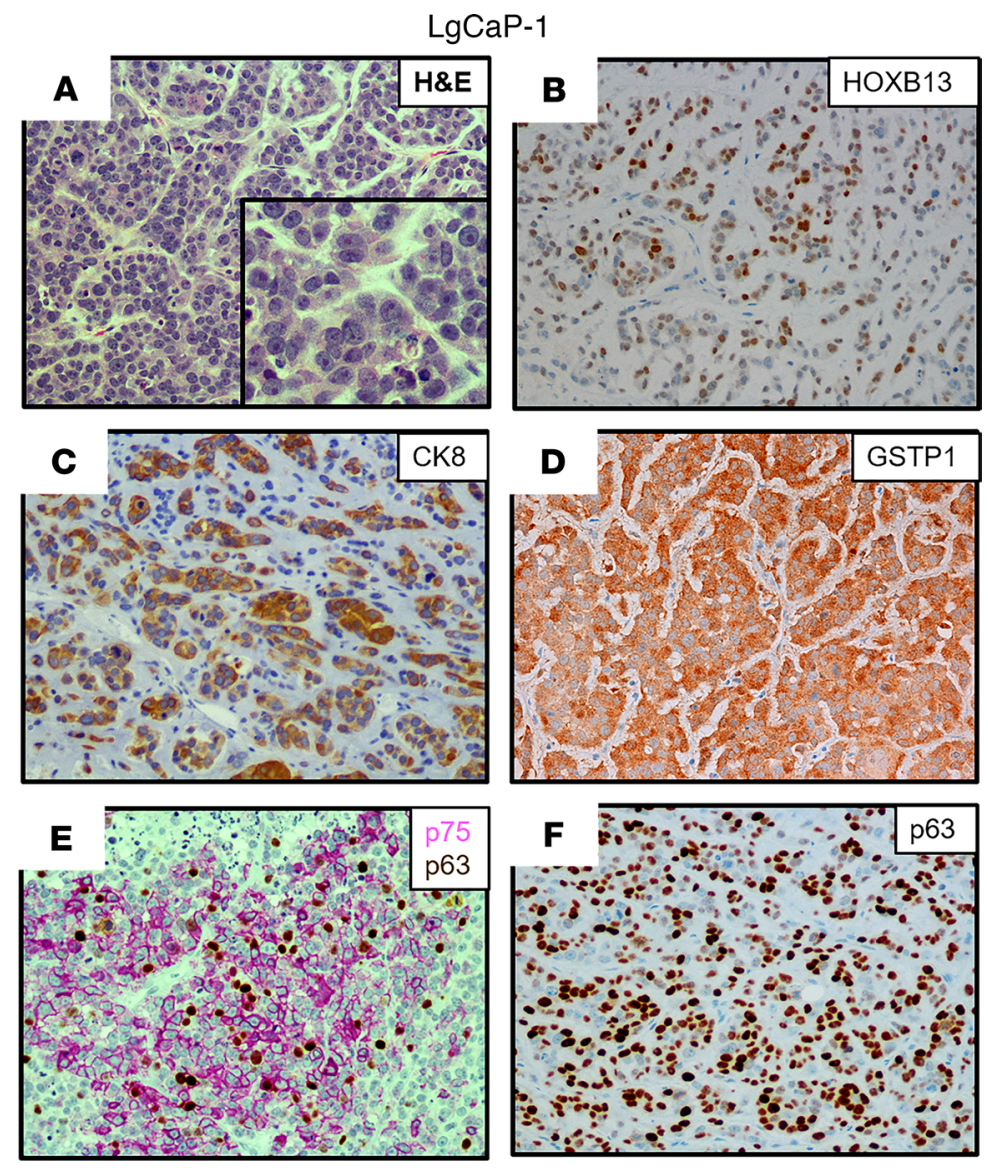

Figure 6. Characteristics of the LgCaP-1 PDX. (A) H\&E histology (original magnification, $\times 200$; inset [original magnification, $\times 400$ ]); (B-E) IHC (original magnification, $\times 200$ ) for (B) HOXB13; (C) CK8; (D) GSTP1; (E) NGFR (pink); and (F) p63 (brown). PDX, patient-derived xenograft; NGFR, nerve growth factor receptor.

Transcriptome-based subtype clustering of PC PDXs. RNA-Seq analysis was performed to allow transcriptome-based clustering of an enlarged PDX series, including the newly developed DN PDXs described in this paper as well as previously characterized PC PDXs from both Johns Hopkins and the University of Washington Prostate Cancer Group $(28,29,43)$. Four of these (i.e., PC-82, CWR22, SkCaP-1, LvCaP-1) are androgen-responsive ARPCs that regress when intact NSG immune-deficient adult male mice bearing these growing PDXs are castrated $(29,50)$. One (i.e., LvCaP-2) is an androgen-responsive AMPC, which regresses when tumor-bearing intact adult male mice are castrated, and 2 (i.e., LvCaP-2R and LuCaP77CR) are CR AMPC grown in castrated hosts $(29,43)$. The remaining 14 PDXs are also CR models grown in castrated hosts. Four (i.e., CWR22-RH, SkCaP-1R, 78CR, and 147CR) are CR ARPCs. Four (i.e., LuCaP 93, 145.1, 145.2, and 173.1) are NEPCs, and the remaining 6 (i.e., BCaP-1, LgCaP-1, LvCap-3, PLNCaP-1, LuCaP 173.2, and LvCaP-1R) are DNPCs. Multidimensional scaling (MDS) was performed based on the expression of a panel of 21 genes (10 gene "NE" and "AR" signatures plus AR; ref. 28). This MDS documents the expected clustering of ARPC versus NEPC PDXs (Figure 10A). The AMPC PDXs demonstrate an intermediate clustering due to the coexpression of AR and NE gene signatures (Figure 10A). In contrast, DNPC PDXs were distinctly clustered from the other phenotypes (Figure 10A).

Androgen receptor promoter hypermethylation differed in DN versus NE PDXs. DNPC and NEPC PDXs showed differential clustering despite neither one expressing AR. To determine whether these transcriptional differences were in part due to differences in AR transcriptional silencing, we performed genome scale and site-specific DNA methylation analyses. Infinium methylation EPIC array studies revealed a region of hypermethylation around the $A R$ transcriptional start site that was present in the NEPC line LuCaP 93, but not in the DNPC lines LvCaP-1, BCaP-1, LgGaP-1, LvCaP-3, or PLNCaP-1 (Figure 10, B and C). As a positive control, AR ${ }^{-}$DU145 


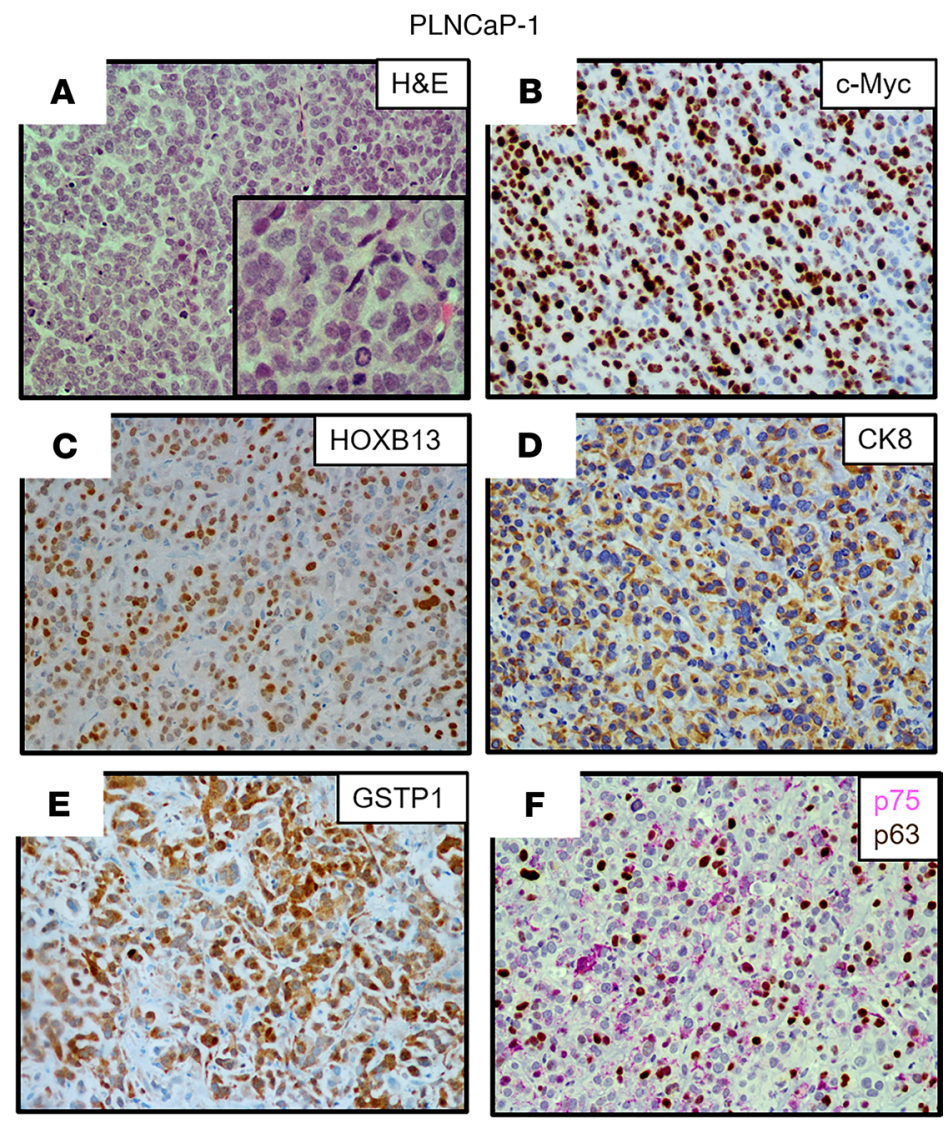

Figure 7. Characteristics of the PLNCaP-1 PDX. (A) H\&E histology (original magnification, $\times 200$; inset [original magnification, $\times 400$ ]). (B-F) IHC (original magnification, $\times 200$ ) for (B) C-MYC; (C) HOXB13; (D) CK8; (E) CSTP1; and (F) NGFR (pink) and p63 (brown). PDX, patient-derived xenograft; NGFR, nerve growth factor receptor.

PC cells were used because they are known to have hypermethylation around the transcriptional start site and expanding into the first exon $(51,52)$. As a negative control, PC3 cells were included $(51,52)$.

These findings were further corroborated by a targeted assessment of DNA methylation of the $A R$ locus using COMPARE-MS (53). Of the 9 ARPCs evaluated, 8 showed minimal to no AR exon 1 DNA hypermethylation (Figure 10D), which is consistent with their high AR expression. Of note, in the 6 evaluated DNs lacking AR expression, no significant $A R$ exon 1 DNA hypermethylation was detected (Figure $10, \mathrm{~B}$ and D). This is in contrast to the 6 NEPC PDXs evaluated, where 5 of the 6 lines showed DNA hypermethylation at this site (Figure 10, $\mathrm{B}$ and $\mathrm{D}$ ), consistent with the lack of $A R$ expression. These results suggest that the NEPC PDXs evaluated here were derived from cells that lost AR expression due to promoter hypermethylation whereas AR was transcriptionally silenced in DNPC PDXs via a different mechanism.

As a potential mechanism for the suppression of the NE phenotype within DN PDXs, a promoter methylation analysis of the 10 NE-related genes described in Figure 3B was performed. This analysis documented that only 4 of the 10 genes (i.e., CHRNB2, PCSK1, ASCL1, and NKX2-1) showed differential methylation consistent with the suppression of the NE phenotype in the DN versus NE PDXs (Supplemental Figure 3). Importantly, in only 1 of the genes (i.e., CHRNB2) was the promoter methylation pattern in the LvCaP-2/-2R amphicrine PDXs, consistent with that expected based on the NE phenotype in LuCaP 93 (Supplemental Figure 4).

\section{Discussion}

Lineage plasticity of PC-initiating cells into a NE phenotype is supported by observations that multiple treatments can induce varying degrees of morphologic and phenotypic NE differentiation of prostate ARPC cells in vitro, including cAMP, IL-6, and serum starvation (54-57). In addition, ARS in ARPC cells can repress such NE differentiation in cell culture models (58-62). Based upon these results, it has been 

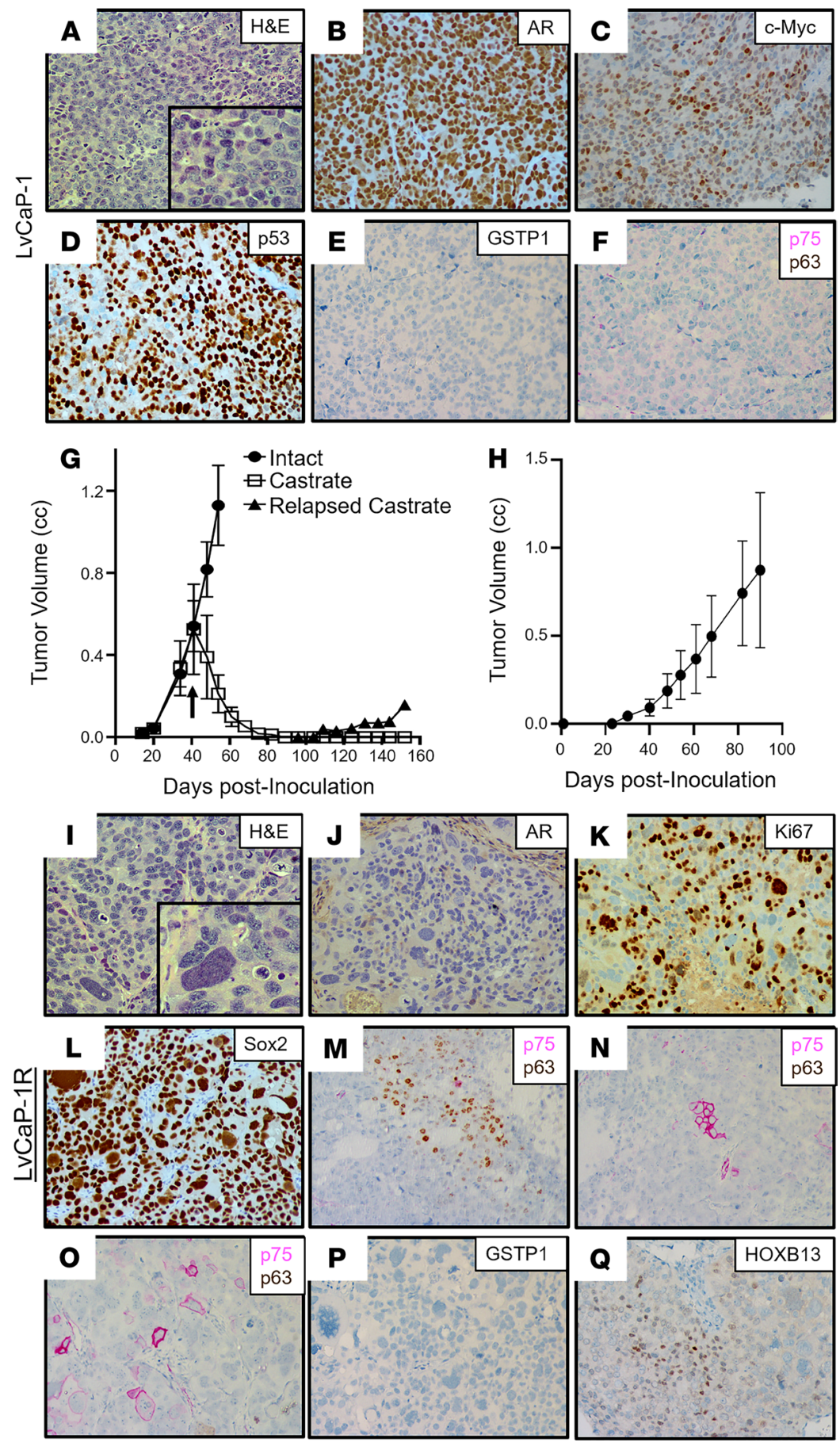

Figure 8. Characteristics of the LvCaP-1 PDX. (A) H\&E histology (original magnification, $\times 200$; inset [original magnification, $\times 400]$ ). (B-F) IHC (original magnification, $\times 200$ ) for (B) AR; (C) HOXB13; (D) p53; (E) GSTP1; and (F) dual staining for NGFR (pink) and p63 (brown). (G) Growth rate in intact and subsequent regression and relapse to castration in NSG male mice $(n=5$ each). Characteristics of the LvCaP-1R PDX. (H) Growth rate in castrated NSG male mice $(n=5$ each); (I) H\&E histology (original magnification, $\times 200$; inset [original magnification, $\times 400$ ]) showing a pleomorphic giant cell. (J-Q) IHC (original magnification, $\times 200$ ) for (J) AR; (K) Ki67; (L) SOX2; (M-0) dual staining for NGFR (pink) and p63 (brown); (P) GSTP1; and (Q) HOXB13. PDX, patient-derived xenograft; NGFR, nerve growth factor receptor.

proposed that ARSi treatment of patients with MCRPC drives ARPCs into a more aggressive and lethal $\mathrm{AR}^{-}$NEPC phenotype $(28,31-34)$. In particular, it has been suggested that such differentiation into NEPC requires the loss of ARS in combination with mutations in PTEN, RB1, and TP53 together with the overexpression of DNMTs, EZH2, and/or SOX2 $(28,31-34,63)$ 
A
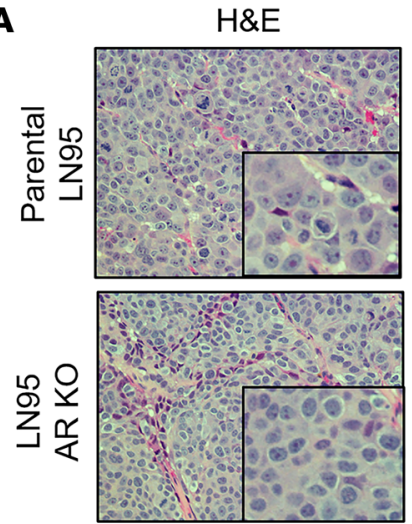

B

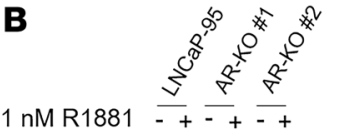

AR-FL

AR-Vs

AR-FL

AR-V7

$\beta$-actin

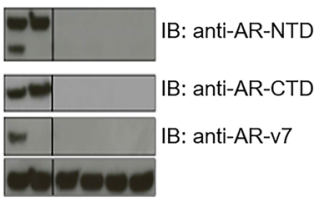

D

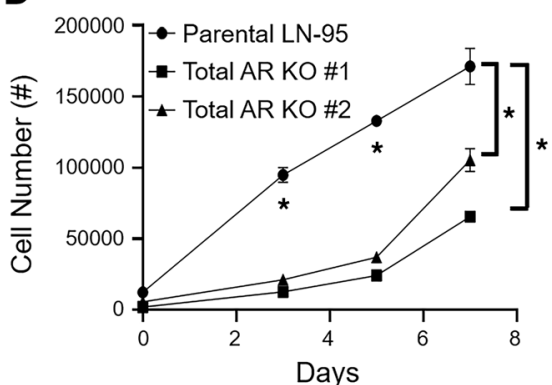

E

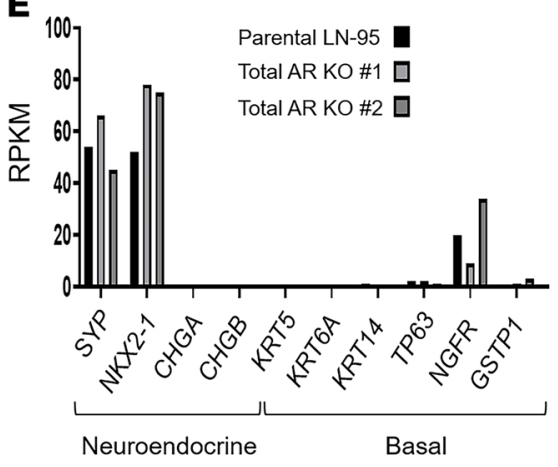

AR
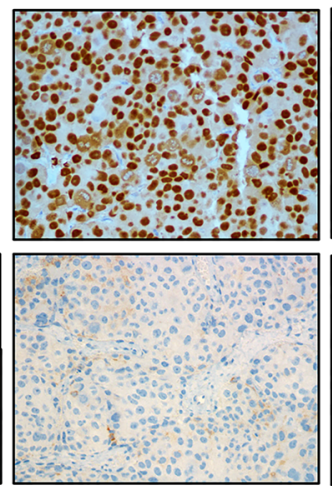

C $\quad \mathrm{LN}-95$

AR-NTD

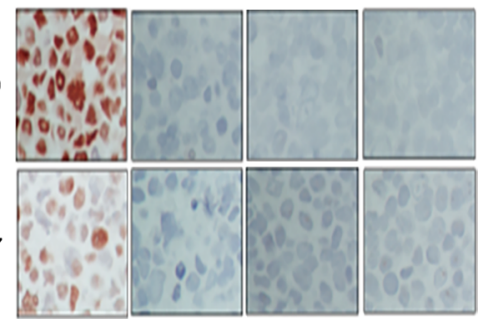

G ${ }^{2.0} 7 \rightarrow$ Parental LN-95

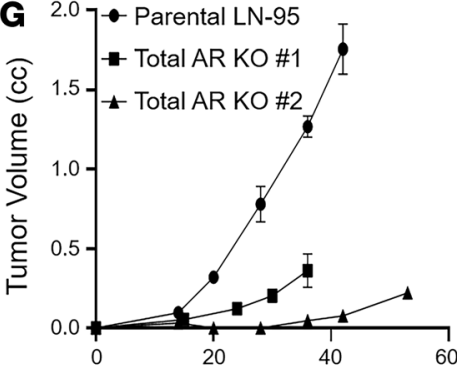

Days post-Inoculation
$\mathbf{F}$

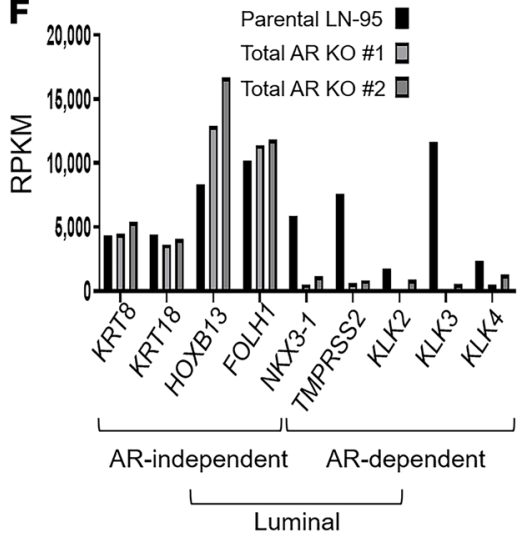

Figure 9. Characterization of LN-95 parental vs. total AR-KO cells. (A) Left panels are the histology (original magnification, $\times 200$; inset [original magnification, $\times 400$ ]); middle panels are the AR protein expression (original magnification, $\times 200$ ); and right panels are the Ki67 expression (original magnification, $\times 200$ ) of the PDXs. (B) Western blot documentation of the successful KO of AR protein in multiple clones of LN-95 cells. (C) IHC (original magnification, $\times 200$ ) staining of parental LN-95 cells expressing both full-length AR (AR-FL) and AR variant 7 (AR-V7) vs. AR ${ }^{-}$PC-3 cells and the AR-KO clones using an N-terminal AR antibody and an AR-V7-specific antibody. (D) In vitro growth of the parental LN-95 cells vs. total AR-KO clones in 10\% CS-FBS media, with asterisks denoting a significant difference at the $P<$ 0.05 level. (E) RNA-Seq-based comparison of the expression of NE-specific and basal-specific genes in total AR-KO clones compared with parental LN-95 cells. (F) RNA-Seq-based comparison of the expression of AR-independent and AR-dependent luminal-specific genes in total AR-KO clones compared with parental LN-95 cells (note the significant difference in the magnitude of the y axis between panels). (G) In vivo growth of the total AR-KO clones vs. the parental LN-95 in castrated hosts. LN-95, LNCaP-95. 


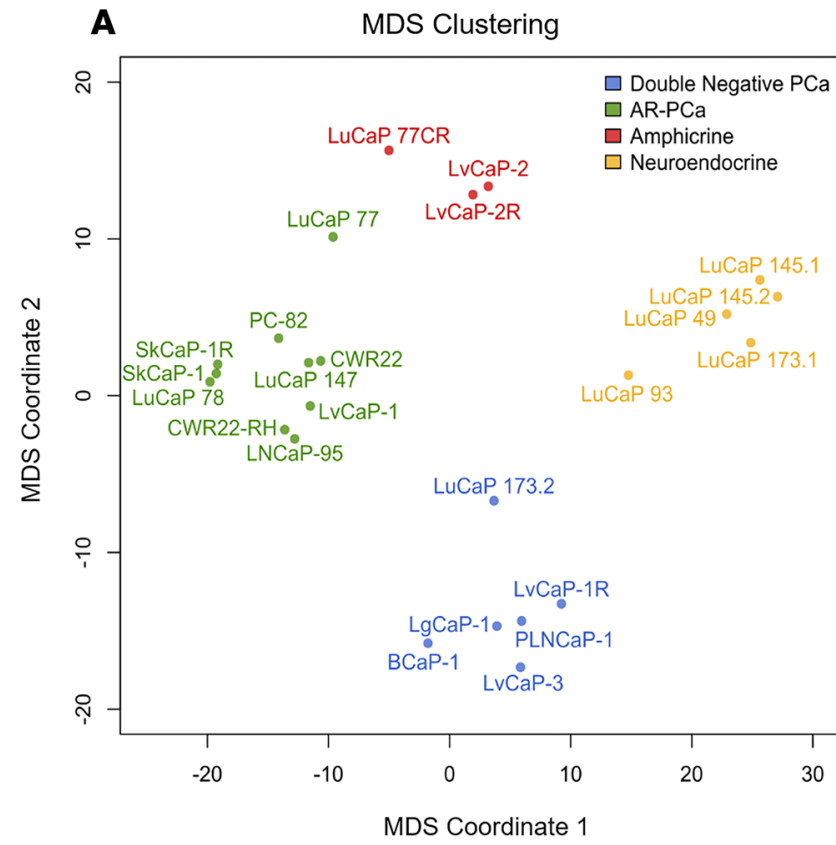

C

Schematic of $A R$ locus and CpG Islands

$A R$

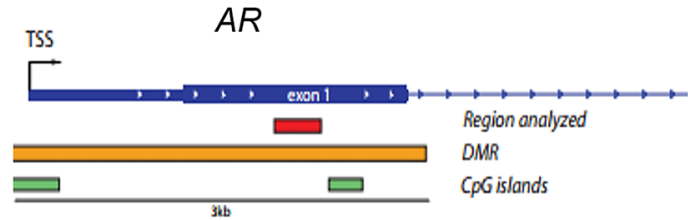

B

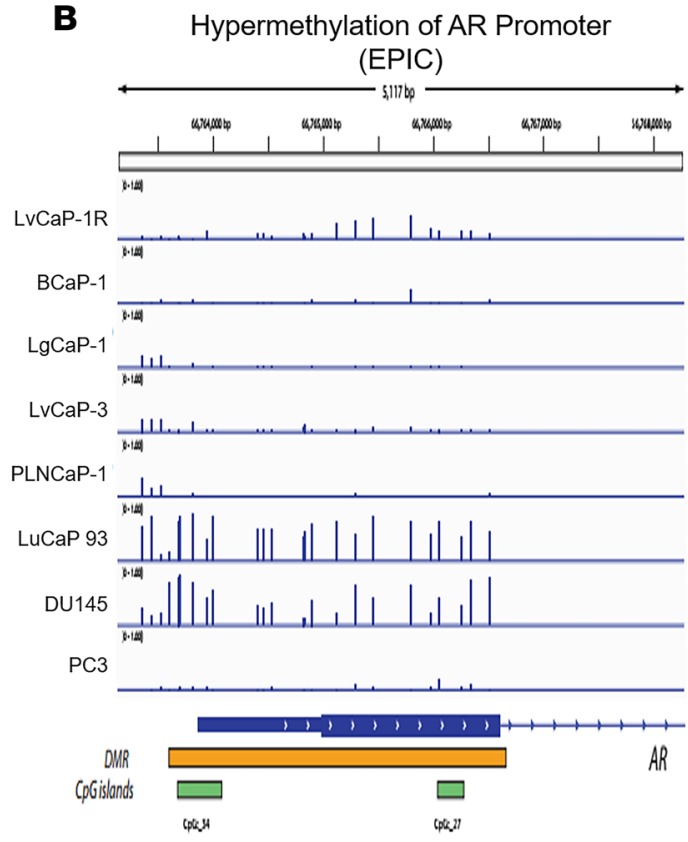

D AR Promoter Methylation Index (COMPARE-MS)

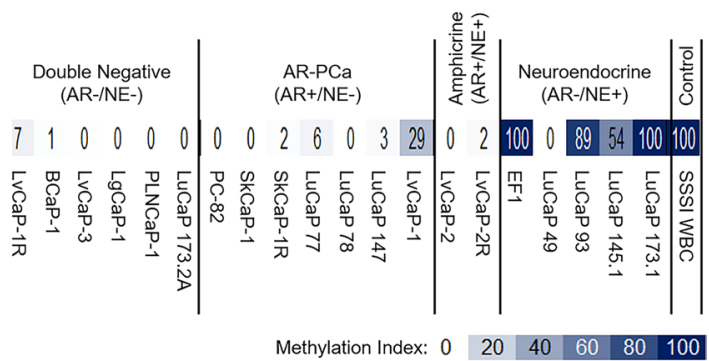

Figure 10. Clustering of lethal mCRPC PDXs and analysis of AR promoter methylation. (A) Clustering of PDX models based on multidimensional scaling. (B) Analysis of methylation levels at the single CpG level using Illumina EPIC arrays reveals hypermethylation of a region encompassing the transcriptional start site and the first exon of $A R$ in LuCaP 93 and DU145 cells. (C) Schematic of the AR locus showing CpG islands, the putative differentially methylated region, and the region interrogated in this COMPARE-MS study. (D) Heat map of methylation indices in the first exon of AR in PDX lines as assessed by COMPARE-MS. mCRPC, metastatic castration-resistant prostate cancer; PDXs, patient-derived xenografts.

The present studies demonstrate that the lack of ARS in combination with these molecular alterations does not always necessitate progression of ARPCs to an ARSi-resistant NEPC phenotype. This conclusion is supported by previous reports. For example, Frigo and McDonnell demonstrated that only incomplete NE differentiation of ARPCs in vitro is produced by the inhibition of ARS with AR antagonists or siRNA-mediated downregulation of AR (62). Additionally, although HSP-90 inhibition downregulates AR expression, it has no effect on NE differentiation (62). In contrast, histone deacetylase (HDAC) inhibitor-induced epigenetic changes can promote such NE differentiation (62). Similarly, ADT is reported to activate the CREB/EZH2 axis, resulting in epigenetic activation of NE differentiation (64). Interestingly, the PDX models used in this study expressed both CREB and EZH2, and yet only a subset of the PDXs that lacked AR expression was NEPC. In addition, LNCaP cells stably transfected with $M Y C N$ phenotypically resemble NEPC with the upregulation of the NE markers and EZH2 coupled with the downregulation of AR and androgen-regulated genes compared with parental cells (30). However, in the PDXs used in our studies, MYCN was highly expressed by BCaP-1, LVCaP-3, and LgCaP-1, and despite no AR expression, these cells did not exhibit NEPC characteristics. Hyperactive mTOR is reported to induce NE differentiation in vitro in LNCaP cells with the concurrent upregulation of IFN regulatory factor 1 and the downregulation of ARS associated with 
the upregulation of CDKN1A (also known as p21) and growth arrest (65). However, all of the ARPDXs reported herein expressed a high level of p21, but only a subset underwent NE differentiation and none were growth arrested. Thus, the present results document that ARSi resistance can occur without ARPC differentiation into a NEPC. These results suggest that in addition to the loss of AR, other molecular changes are needed for NEPC differentiation. There are several candidate changes for such NEPC drivers, including the downregulation of REST and YAP, in addition to the upregulation of $A S C L 1$, a transcription factor important in neuronal development $(15,40,66)$. Of note, it is significant that the DNPC PDXs did not downregulate REST and YAP1 or upregulate ASCL1 (Figure 3A); whereas, the NEPC PDXs did undergo these changes (Figure 3A).

Collectively, these results document that ARSi resistance occurs in both NEPC and DNPC via a loss of AR expression. In NEPC, this loss was frequently associated with hypermethylation and silencing of the AR promoter, which is consistent with previous studies supporting their derivation from ARPC cells via lineage plasticity $(28,31-34)$. In contrast, hypermethylation of the AR promoter was not detected in the current DNPC PDX series. In addition, differential promoter hypermethylation does not provide a simple explanation for the suppression of NE-related gene expression in the different phenotypes.

Importantly, all of the DNPC PDXs described herein had a mixture of malignant cells that heterogeneously expressed basal markers either alone or in combination with luminal markers. This phenotypic heterogeneity was present despite all cancer cells within each PDX having identical genetic driver mutations. There are at least 2 potential mechanisms to explain this phenomenon and the emergence of ARSi-resistant DNPC: (a) an initially $\mathrm{AR}^{+}$cancer-initiating cell loses AR expression under ADT and acquires this phenotypic heterogeneity via lineage plasticity (i.e., adaptation); or (b) an $\mathrm{AR}^{-}$prostate progenitor cell is the cancer-initiating cell and gives rise to malignant progeny heterogeneously expressing various combinations of basal and luminal markers (i.e., selection). In this second scenario, no promoter hypermethylation-dependent silencing of AR would be necessary for selective outgrowth of these DN cells under extreme androgen deprivation. Presently, these PDX models are being utilized to resolve whether adaption versus selection is the mechanism for the emergence of ARSi-resistant DNPC. Earlier studies demonstrated that PC with a luminal phenotype could be derived from genetically manipulated primary human benign prostate basal cells, suggesting that histology does not necessarily correlate with cell of origin (67).

Regardless of whether adaption or selection is responsible, approximately one-third of ARSi-resistant cancers are either DNPCs or NEPCs that lack AR expression, and the frequencies of such $\mathrm{AR}^{-} \mathrm{PCs}$ are increasing (26). Thus, there is an urgent need for the development of therapies that do not depend upon AR activity for their efficacy $(28,68,69)$. Thus, the PDXs characterized in the present report provide a credentialed platform for such drug development.

\section{Methods}

Detailed procedures describing PDX establishment, cell culture, proliferation assays, cytogenetic, genetic and epigenetic characterization, plasmid construction and transfection of CRISPR/Cas9 vectors, isolation of clonal cell lines by FACS, RNA-Seq, DNA sequencing, methylation, Western blot, IHC, animal studies, and statistical analyses are included in the Supplemental Methods.

Study approval. Tissue collection for research was approved by the Johns Hopkins University School of Medicine IRB. Tumor specimens were acquired from patients with mCRPC who signed informed consent. All animal procedures were approved by the Johns Hopkins University School of Medicine Institutional Animal Care and Use Committee.

Data availability. The RNA-Seq data for this publication has been deposited in NCBI's Gene Expression Omnibus and are accessible through accession number GSE160393 for the raw and mouse-gene subtracted PDX data, and GSE131985 for the LN-95 and AR-KO cells.

\section{Author contributions}

WNB, YZ, and JTI conceived and designed the study. YZ, IMC, SLD, LA, RAP, BH, RC, AKM, and SLZ developed the methodology. YZ, IMC, SLD, LA, RAP, BH, RC, AKM, SLZ, JEH, and AMDM acquired the data (e.g., provided animals, acquired patients, provided facilities). WNB, YZ, IMC, SLD, LA, RAP, BH, RC, AKM, SLZ, JEH, JL, AMDM, EC, JX, SY, MCH, PSN, WGN, WBI, and JTI analyzed and interpreted the data (e.g., statistical analysis, bioinformatics, computational analysis). WNB, YZ, IMC, 
SLD, LA, RAP, BH, RC, AKM, SLZ, JEH, JL, AMDM, EC, JZ, SY, MCH, PSN, WGN, WBI, and JTI wrote, reviewed, and/or revised the manuscript. YZ, IMC, JEH, EC, and JL provided administrative, technical, or material support (e.g., reporting or organizing data, constructing databases, rapid autopsies, sharing resources). WNB and JTI supervised the study.

\section{Acknowledgments}

We are grateful to the patients and their families who participated in the Legacy Gift Rapid Autopsy program at Johns Hopkins. We acknowledge the following sources of financial support: Movember Foundation Global Action Plan program for development of PC xenografts to model human PC (to JTI); Prostate Cancer Foundation Young Investigator Award (to WNB) and Challenge Awards (to SY, PSN); Patrick C. Walsh Prostate Cancer Research Fund (to WNB); Department of Defense Prostate Cancer Research Program Awards W81XWH-16-1-0410 and W81XWH-18-1-0348 (to JTI), W81XWH-18-1-0347 and W81XWH-17-1-0415 (to PSN), W81XWH-17-1-0528 (to WNB), W81XWH-20-1-0111 (to MCH), W81XWH-10-2-0045 and W82XWH-10-2-0056 PCRP Prostate Cancer Biorepository Network (to AMDM); Safeway Foundation (to $\mathrm{MCH}$ ); Commonwealth Foundation Award (to SY); NIH-Prostate SPORE grant P50 CA058236 (to JTI and SY); NIH grant P50 CA058236 (to AMD for the Pathology Core); NIH-Prostate SPORE grant P50 CA097186 (to IMC, BH, MCH, PSN); NIH R01 CA255259 (to WNB), R01 CA185297 (to JL), P01CA163227 (to PSN), R01 CA234715 (to PSN), and P30 CA015704 (to IMC and PSN); and the NIH-SKCCC Cancer Center Support Grant award P30 CA006973, which provides support for the Oncology Tissue Services, Animal Resources, Cell Imaging, Rapid Autopsy Core, Genetic Resources, and Experimental and Computational Genomics Core Facilities.

Address correspondence to: John. T. Isaacs, Johns Hopkins School of Medicine, 1650 Orleans St., CRB-I, Rm 1M4, Baltimore, Maryland 21287, USA. Phone: 410.955.7777; Email: isaacjo@jhmi.edu.

1. Isaacs JT, Coffey DS. Etiology and disease process of benign prostatic hyperplasia. Prostate Suppl. 1989;2:33-50.

2. Moad M, et al. Multipotent basal stem cells, maintained in localized proximal niches, support directed long-ranging epithelial flows in human prostates. Cell Rep. 2017;20(7):1609-1622.

3. Berges RR, et al. Implication of cell kinetic changes during the progression of human prostatic cancer. Clin Cancer Res. 1995;1(5):473-480.

4. Litvinov IV, et al. Is the Achilles' heel for prostate cancer therapy a gain of function in androgen receptor signaling? J Clin Endocrinol Metab. 2003;88(7):2972-2982.

5. Kurita T, et al. Paracrine regulation of apoptosis by steroid hormones in the male and female reproductive system. Cell Death Differ. 2001;8(2):192-200.

6. Wang Y, et al. Cell differentiation lineage in the prostate. Differentiation. 2001;68(4-5):270-279.

7. Bonkhoff H, Remberger K. Differentiation pathways and histogenetic aspects of normal and abnormal prostatic growth: a stem cell model. Prostate. 1996;28(2):98-106.

8. Hudson DL, et al. Epithelial cell differentiation pathways in the human prostate: identification of intermediate phenotypes by keratin expression. J Histochem Cytochem. 2001;49(2):271-278.

9. Pignon JC, et al. p63-expressing cells are the stem cells of developing prostate, bladder, and colorectal epithelia. Proc Natl Acad Sci U S A. 2013;110(20):8105-8110.

10. Bonkhoff H, et al. Endocrine-paracrine cell types in the prostate and prostatic adenocarcinoma are postmitotic cells. Hum Pathol. 1995;26(2):167-170.

11. Xue Y, et al. Prostatic neuroendocrine cells have a unique keratin expression pattern and do not express Bcl-2: cell kinetic features of neuroendocrine cells in the human prostate. Am J Pathol. 1997;151(6):1759-1765.

12. Rumpold H, et al. Neuroendocrine differentiation of human prostatic primary epithelial cells in vitro. Prostate. 2002;53(2):101-108.

13. Litvinov IV, et al. Low-calcium serum-free defined medium selects for growth of normal prostatic epithelial stem cells. Cancer Res. 2006;66(17):8598-8607.

14. Yu X, et al. SOX2 expression in the developing, adult, as well as, diseased prostate. Prostate Cancer Prostatic Dis. 2014;17(4):301-309.

15. Cheng $\mathrm{S}$, et al. The expression of YAP1 is increased in high-grade prostatic adenocarcinoma but is reduced in neuroendocrine prostate cancer. Prostate Cancer Prostatic Dis. 2020;23(4):661-669.

16. Papandreou CN, et al. Reversal of the low-affinity neurotrophin receptor stromal-epithelial expression pattern between benign and malignant human prostate. Urol Oncol. 1998;4(6):210-217.

17. Johng D, et al. HOXB13 interaction with MEIS1 modifies proliferation and gene expression in prostate cancer. Prostate. 2019;79(4):414-424.

18. McMullin RP, et al. A FOXA1-binding enhancer regulates Hoxb13 expression in the prostate gland. Proc Natl Acad Sci U S A. 2010;107(1):98-103.

19. Bostwick DG, et al. Prostate specific membrane antigen expression in prostatic intraepithelial neoplasia and adenocarcinoma: a study of 184 cases. Cancer. 1998;82(11):2256-2261.

20. Ewing CM, et al. Germline mutations in HOXB13 and prostate-cancer risk. N Engl J Med. 2012;366(2):141-149. 
21. Donjacour AA, Cunha GR. Assessment of prostatic protein secretion in tissue recombinants made of urogenital sinus mesenchyme and urothelium from normal or androgen-insensitive mice. Endocrinology. 1993;132(6):2342-2350.

22. Evans MJ, et al. Noninvasive measurement of androgen receptor signaling with a positron-emitting radiopharmaceutical that targets prostate-specific membrane antigen. Proc Natl Acad Sci U S A. 2011;108(23):9578-9582.

23. Gurel B, et al. NKX3.1 as a marker of prostatic origin in metastatic tumors. Am J Surg Pathol. 2010;34(8):1097-1105.

24. Vander Griend DJ, et al. Conversion of androgen receptor signaling from a growth suppressor in normal prostate epithelial cells to an oncogene in prostate cancer cells involves a gain of function in c-Myc regulation. Int J Biol Sci. 2014;10(6):627-642.

25. Antony L, et al. Androgen receptor (AR) suppresses normal human prostate epithelial cell proliferation via AR/ $\beta$-catenin/ TCF-4 complex inhibition of c-MYC transcription. Prostate. 2014;74(11):1118-1131.

26. Gao S, et al. Androgen receptor tumor suppressor function is mediated by recruitment of retinoblastoma protein. Cell Rep 2016;17(4):966-976.

27. Watson PA, et al. Emerging mechanisms of resistance to androgen receptor inhibitors in prostate cancer. Nat Rev Cancer. 2015;15(12):701-711.

28. Bluemn EG, et al. Androgen receptor pathway-independent prostate cancer is sustained through FGF signaling. Cancer Cell. 2017;32(4):474-489.

29. Zhu Y, et al. Role of androgen receptor splice variant-7 (AR-V7) in prostate cancer resistance to 2nd-generation androgen receptor signaling inhibitors. Oncogene. 2020;39(45):6935-6949.

30. Beltran H, et al. Molecular characterization of neuroendocrine prostate cancer and identification of new drug targets. Cancer Discov. 2011;1(6):487-495.

31. Beltran H, et al. Divergent clonal evolution of castration-resistant neuroendocrine prostate cancer. Nat Med. 2016;22(3):298-305.

32. Ku SY, et al. Rb1 and Trp53 cooperate to suppress prostate cancer lineage plasticity, metastasis, and antiandrogen resistance. Science. 2017;355(6320):78-83.

33. $\mathrm{Mu}$ P, et al. SOX2 promotes lineage plasticity and antiandrogen resistance in TP53- and RB1-deficient prostate cancer. Science. 2017;355(6320):84-88.

34. Park JW, et al. Reprogramming normalhuman epithelial tissues to a common, lethal neuroendocrine cancer lineage. Science. 2018;362(6410):91-95.

35. Wang HT, et al. Neuroendocrine Prostate Cancer (NEPC) progressing from conventional prostatic adenocarcinoma: factors associated with time to development of NEPC and survival from NEPC diagnosis-a systematic review and pooled analysis. J Clin Oncol. 2014;32(30):3383-3390.

36. Johnson BE, et al. Retention of chromosome 3 in extrapulmonary small cell cancer shown by molecular and cytogenetic studies. J Natl Cancer Inst. 1989;81(16):1223-1228.

37. van Bokhoven A, et al. Molecular characterization of human prostate carcinoma cell lines. Prostate. 2003;57(3):205-225.

38. Mertz KD, et al. Molecular characterization of TMPRSS2-ERG gene fusion in the NCI-H660 prostate cancer cell line: a new perspective for an old model. Neoplasia. 2007;9(3):200-206.

39. Cardoso M, et al. Oncogenic mechanisms of HOXB13 missense mutations in prostate carcinogenesis. Oncoscience. 2016;3(910):288-296.

40. Zhang X, et al. SRRM4 expression and the loss of REST activity may promote the emergence of the neuroendocrine phenotype in castration-resistant prostate cancer. Clin Cancer Res. 2015;21(20):4698-4708.

41. Prendeville S, et al. Prostate carcinoma with amphicrine features: further refining the spectrum of neuroendocrine differentiation in tumours of primary prostatic origin? Histopathology. 2017;71(6):926-933.

42. Bellur S, et al. Evolving concepts in prostatic neuroendocrine manifestations: from focal divergent differentiation to amphicrine carcinoma. Hum Pathol. 2019;85:313-327.

43. Nguyen HM, et al. LuCaP prostate cancer patient-derived xenografts reflect the molecular heterogeneity of advanced disease and serve as models for evaluating cancer therapeutics. Prostate. 2017;77(6):654-671.

44. Yegnasubramanian S, et al. Hypermethylation of CpG islands in primary and metastatic human prostate cancer. Cancer Res. 2004;64(6):1975-1986

45. Wang J, et al. Symmetrical and asymmetrical division analysis provides evidence for a hierarchy of prostate epithelial cell lineages. Nat Commun. 2014;5:4758.

46. Haffner MC, et al. Tracking the clonal origin of lethal prostate cancer. J Clin Invest. 2013;123(11):4918-4922.

47. Pflug BR, et al. Caveolin expression is decreased following androgen deprivation in human prostate cancer cell lines. Prostate. 1999;40(4):269-273.

48. Attard G, et al. Clinical and biochemical consequences of CYP17A1 inhibition with abiraterone given with and without exogenous glucocorticoids in castrate men with advanced prostate cancer. J Clin Endocrinol Metab. 2012;97(2):507-516.

49. Yang YC, et al. Targeting androgen receptor activation function-1 with EPI to overcome resistance mechanisms in castration-resistant prostate cancer. Clin Cancer Res. 2016;22(17):4466-4477.

50. Isaacs JT, et al. Adaptive auto-regulation of androgen receptor provides a paradigm shifting rationale for bipolar androgen therapy (BAT) for castrate resistant human prostate cancer. Prostate. 2012;72(14):1491-1505.

51. Jarrard DF, et al. Methylation of the androgen receptor promoter CpG island is associated with loss of androgen receptor expression in prostate cancer cells. Cancer Res. 1998;58(23):5310-5314.

52. Nakayama T, et al. Epigenetic regulation of androgen receptor gene expression in human prostate cancers. Lab Invest. 2000;80(12):1789-1796.

53. Yegnasubramanian S, et al. Combination of methylated-DNA precipitation and methylation-sensitive restriction enzymes (COMPARE-MS) for the rapid, sensitive and quantitative detection of DNA methylation. Nucleic Acids Res. 2006;34(3):e19.

54. Bang YJ, et al. Terminal neuroendocrine differentiation of human prostate carcinoma cells in response to increased intracellular cyclic AMP. Proc Natl Acad Sci U S A. 1994;91(12):5330-5334.

55. Kim J, et al. Involvement of cholesterol-rich lipid rafts in interleukin-6-induced neuroendocrine differentiation of LNCaP prostate cancer cells. Endocrinology. 2004;145(2):613-619.

56. Wang Q, et al. Interleukin-6 inhibits the growth of prostate cancer xenografts in mice by the process of neuroendocrine 
differentiation. Int J Cancer. 2004;111(4):508-513.

57. Pinski J, et al. Genistein-induced neuroendocrine differentiation of prostate cancer cells. Prostate. 2006;66(11):1136-1143.

58. Gao N, et al. The role of hepatocyte nuclear factor-3 alpha (Forkhead Box A1) and androgen receptor in transcriptional regulation of prostatic genes. Mol Endocrinol. 2003;17(8):1484-1507.

59. Yang Y, et al. Differential expression of cytokeratin mRNA and protein in normal prostate, prostatic intraepithelial neoplasia, and invasive carcinoma. Am J Pathol. 1997;150(2):693-704.

60. Jongsma J, et al. Androgen deprivation of the PC-310 [correction of prohormone convertase-310] human prostate cancer model system induces neuroendocrine differentiation. Cancer Res. 2000;60(3):741-748.

61. Zhang XQ, et al. Receptor protein tyrosine phosphatase alpha signaling is involved in androgen depletion-induced neuroendocrine differentiation of androgen-sensitive LNCaP human prostate cancer cells. Oncogene. 2003;22(43):6704-6716

62. Frigo DE, McDonnell DP. Differential effects of prostate cancer therapeutics on neuroendocrine transdifferentiation. Mol Cancer Ther. 2008;7(3):659-669.

63. Ellis L, Loda M. LSD1: A single target to combat lineage plasticity in lethal prostate cancer. Proc Natl Acad Sci U S A. 2018;115(18):4530-4531.

64. Zhang Y, et al. Androgen deprivation promotes neuroendocrine differentiation and angiogenesis through CREB-EZH2-TSP1 pathway in prostate cancers. Nat Commun. 2018;9(1):4080.

65. Kanayama M, et al. Hyperactive mTOR induces neuroendocrine differentiation in prostate cancer cell with concurrent up-regulation of IRF1. Prostate. 2017;77(15):1489-1498.

66. Clegg N, et al. Molecular characterization of prostatic small-cell neuroendocrine carcinoma. Prostate. 2003;55(1):55-64.

67. Goldstein AS, et al. Identification of a cell of origin for human prostate cancer. Science. 2010;329(5991):568-571.

68. Denmeade SR, Isaacs JT. Engineering enzymatically activated "molecular grenades" for cancer. Oncotarget. 2012;3(7):666-667.

69. Brennen WN, et al. Targeting carcinoma-associated fibroblasts within the tumor stroma with a fibroblast activation protein-activated prodrug. J Natl Cancer Inst. 2012;104(17):1320-1334. 